Research Article

\title{
CNOP-P-Based Parameter Sensitivity Analysis for North Atlantic Oscillation in Community Earth System Model Using Intelligence Algorithms
}

\author{
Bin Mu, ${ }^{1}$ Jing Li, ${ }^{1}$ Shijin Yuan $\mathbb{D}^{1},{ }^{1}$ Xiaodan Luo, ${ }^{1}$ and Guokun Dai ${ }^{2}$ \\ ${ }^{1}$ Department of Software Engineering, Tongji University, Shanghai, China \\ ${ }^{2}$ Department of Atmospheric and Oceanic Sciences \& Institute of Atmospheric Sciences, Fudan University, Shanghai, China \\ Correspondence should be addressed to Shijin Yuan; yuanshijin2003@163.com
}

Received 28 September 2019; Revised 19 July 2020; Accepted 24 September 2020; Published 15 October 2020

Academic Editor: Hisayuki Kubota

Copyright (C) 2020 Bin Mu et al. This is an open access article distributed under the Creative Commons Attribution License, which permits unrestricted use, distribution, and reproduction in any medium, provided the original work is properly cited.

Model error, which results from model parameters, can cause the nonnegligible uncertainty in the North Atlantic Oscillation (NAO) simulation. Conditional nonlinear optimal perturbation related to parameter (CNOP-P) is a powerful approach to investigate the range of uncertainty caused by model parameters under a specific constraint. In this paper, we adopt intelligence algorithms to implement the CNOP-P method and conduct the sensitivity analysis of parameter combinations for NAO events in the Community Earth System Model (CESM). Among 28 model parameters of the atmospheric component, the most sensitive parameter combination for the $\mathrm{NAO}^{+}$consists of parameter for deep convection (cldfrc_dp1), minimum relative humidity for low stable clouds (cldfrc_rhminl), and the total solar irradiance (solar_const). As for the $\mathrm{NAO}^{-}$, the parameter set that can trigger the largest variation of the NAO index (NAOI) is comprised of the constant for evaporation of precip (cldwat_conke), characteristic adjustment time scale ( $\left.h k c o n v \_c m f t a u\right)$, and the total solar irradiance (solar_const). The most prominent uncertainties of the NAOI ( $\triangle \mathrm{NAOI}$ ) caused by these two combinations achieve 2.12 for $\mathrm{NAO}^{+}$and -2.72 for $\mathrm{NAO}^{-}$, respectively. In comparison, the maximum level of the NAOI variation resulting from single parameters reaches 1.45 for $\mathrm{NAO}^{+}$and -1.70 for $\mathrm{NAO}^{-}$. It is indicated that the nonlinear impact of multiple parameters would be more intense than the single parameter. These results present factors that are closely related to NAO events and also provide the direction of optimizing model parameters. Moreover, the intelligence algorithms adopted in this work are proved to be adequate to explore the nonlinear interaction of parameters on the model simulation.

\section{Introduction}

North Atlantic Oscillation (NAO) has the most prominent seesaw-type oscillation in atmospheric circulation between subtropical high and subpolar low [1]. In recent decades, NAO presents the characteristic of abnormal and frequently changed, especially for winter NAO [2]. Recent research indicates that the $\mathrm{NAO}^{+}\left(\mathrm{NAO}^{-}\right)$leads to increased (reduced) temperature [3], and extremely cold weather along with snowstorms in Europe is closely associated with $\mathrm{NAO}^{-}$in a large-scale NAO circulation $[4,5]$. The low-frequency change and seasonal features of NAO have a significant impact on the global climate $[6,7]$, thus estimating the uncertainty of NAO simulation and prediction has practical implications [8].
NAO is a complicated nonlinear system of atmosphere, and it is widely considered to be mainly triggered by Rossby wave breaking [9]. Beyond that, the origins of the NAO fluctuation are complex and varied, including sea surface temperature (SST) anomalies in both tropics and extratropics, extreme stratospheric events [10], tropical atmospheric heat anomalies [11], and intensity of geomagnetic activity [12]. Several models have been developed to describe the nonlinear scale interaction and the life cycle of NAO $[13,14]$, such as the CERFACS forecast system [15], CMCCINGV [16], and Community Earth System Model (CESM) [17]. However, the climate simulation using dynamic models would be influenced by both initial condition errors and model errors [18], which have become two major 
predictability problems [19]. Thereinto, model error is considered as one of the main reasons that cause inaccurate modeling, and it would make the uncertainty exist in the NAO simulation and prediction $[20,21]$. Previous researches have pointed out that the prediction skills can be improved by reducing errors of sensitive parameters $[22,23]$.

The first step in optimizing the model via parameter correction is to determine the sensitive parameters and evaluate corresponding sensitivities. Sensitivity analysis is a method to quantify the sensitivity according to the uncertainty of objective function, and it can help us to identify critical relations between variables and prediction. In previous studies, a few approaches have been adopted to explore the sensitivity of physical parameters in numerical models [24]. The hybrid approach, which is coupled with Morris screening method, response surface method, and RSMSobol method, has been successfully applied to evaluate the impact of global parameters in the distributed time-variant gain model (DTVGM), thereby providing a scheme to improve parameter estimation of the model [25]. Griensven et al. have adopted a novel sampling strategy combined with Latin-hypercube and one-factor-at-a-time sampling in a hydrological model to conduct global sensitivity analysis for parameters with a limited number [26]. In the research on soil moisture, the global sensitivity analysis based on the advanced integral equation model highlights the quantitative and systematic evaluation of parameter sensitivities [27]. Although the uncertainties in these climate simulations have been recognized and the corresponding sensitivity analysis schemes with single parameters have been performed, current studies may overlook the dependencies and interactions among these parameters and have not simultaneously considered multiple parameters. Besides, sensitive parameters in their researches are selected based on experience, and potential sensitive parameters may be missed out.

As mentioned above, previous studies have investigated the uncertainty owing to model parameters in simulating several climate events. However, the research on the estimation of model error for the NAO is almost in blank. In this paper, we aim to explore the maximal extent of uncertainty caused by sensitive parameters in the NAO simulation using CESM. CESM is one of the leading earth system frameworks [28] to simulate the evolution of the NAO fluctuation, and a flood of achievements have obtained in numerical modeling of NAO using CESM [29, 30]. The conditional nonlinear optimal perturbation related to parameter (CNOP-P) method, which was introduced by $\mathrm{Mu}$ et al. in 2010 [31], is utilized in this work. Using the CNOP-P method, the range of model uncertainty and parameter errors are solved under a reasonable constraint at prediction time. The CNOP-P method has been applied in multiple fields in geography, such as El Niño-Southern Oscillation (ENSO) [32], grassland ecosystems [33], terrestrial ecosystems [34], solid moisture [35], double-gyre variation [36], and net primary production (NPP) [37]. In the predictability study of ENSO, it is indicated that the bias of model parameters obtained by the CNOP-P method yields a large prediction error [32]. In addition, the extent of grassland ecosystem variation triggered by the CNOP-P-type perturbation is much higher than that caused by linear-type vectors [33]. For double-gyre variation in the Regional Ocean Modeling System (ROMS), the effect of sensitive parameters (combinations) on model simulation is verified using an improved simulated annealing algorithm [36]. These research results proved that the CNOP-P method is appropriate for the investigation of the parameter sensitivity in numerical models, especially for multiparameter combinations.

According to the definition, the parameter perturbations obtained by CNOP-P have the most significant effect on prediction results, and it can be tackled as the constrained extreme value problem. To implement the CNOP-P approach, the adjoint-based algorithm is generally used; for instance, the Spectral Projected Gradient (SPG) method [38]. This kind of algorithm has its limitations because it relies on the gradient information provided by the adjoint model, and it is not applicable for models that do not have an adjoint model, like CESM. Thus, we adopt adjoint-free algorithms to implement CNOP-P and select the variation of $\mathrm{NAO}$ index ( $\triangle \mathrm{NAOI})$ as the objective function to evaluate impacts. After control experiments, 28 potential sensitive parameters are chosen from all the parameters of atmospheric component and ice component. These potential sensitive parameters are grouped into combinations with two or three parameters. Then two intelligence algorithms are applied to further analyze the impact of model parameters. Particle swarm optimization (PSO) is adopted for single parameters and double-parameter combinations. PSO is a kind of swarm intelligence algorithm to solve the nondeterministic polynomial hard (NP-hard) problem and has been widely adopted in solving CNOP-I-type problems [39-41]. As for the combinations that contain more parameters, we utilize the method called the covariance matrix adaptation evolution strategy (CMA-ES) to achieve fast convergence. CMA-ES is commonly used for global optimization problems, such as the parameter adjustment in the neural network [42], large-scale overlapping problems [43], and feature weighting problems [44]. The objective of this work is (1) find out which single parameter or parameter combination can cause the maximal extent of uncertainty for two types of NAO events, and record their values, (2) investigate how much of uncertainties that the single parameter or parameter combination in (1) can achieve, and (3) explore the magnitude of parameter perturbations that can cause the extreme NAO events. The results demonstrate that even a tiny parameter error may cause a large margin of model error. Besides, the comprehensive influence of parameter combinations is greater than that of the single parameter. The most sensitive parameter combinations are parameter for deep convection cloud fraction ( $c l d f r c \_d p 1$ ), minimum relative humidity for low stable clouds (cldfrc_rhminl), the total solar irradiance (solar_const), and the constant for evaporation of precip (cldwat_conke), characteristic adjustment time scale ( $\left.h k c o n v \_c m f t a u\right)$, the total solar irradiance (solar_const) for $\mathrm{NAO}^{+}$and $\mathrm{NAO}^{-}$, respectively. Our study is also conducive to parameter optimization to improve forecasting skills in future work. 
The structure of this paper is organized as follows: Section 2 describes the basic information of CESM, the principle of PSO algorithm, and the strategies in CMA-ES. Experiments and results are displayed in Section 3. This paper ends with discussions and conclusions in Section 4.

\section{Materials and Methods}

2.1. CESM. As a new generation of the large-scale unitycoupled climate model, CESM provides efficient simulation for global climate and natural variability, and it has been widely used in the research of the midlatitude ocean variability [45], interannual variability of summer surface air temperature [46], and air quality [47], etc. Meanwhile, several scholars also utilized CESM to simulate the evolution of NAO with related factors, such as temperature [30]. The CESM assembles atmosphere, ocean, land, and sea-ice component models coupled through a coupler. The corresponding atmospheric component is derived from the Community Atmosphere Model (CAM), and the land component is the Community Land Model (CLM).

2.2. CNOP-P. CNOP is a practical approach to study the initial error (CNOP-I) and the model parameter error (CNOP-P), which have the largest effect on prediction results. The research of CNOP-I is on the basis of the condition that prediction errors are entirely from the initial state. However, the model parameters, which are determined by observation experiments, can also cause the prediction error. Therefore, such kind of predictability problem was raised to explore the impact due to model parameters.

We can assume that the basic state $\mathbf{U}$ satisfies the following partial differential equation:

$$
\left\{\begin{array}{l}
\frac{\partial U}{\partial T}=F(\mathbf{U}, \mathbf{P}), \\
\left.\mathbf{U}\right|_{t=0}=\mathbf{U}_{0}
\end{array}\right.
$$

where $F$ denotes the nonlinear propagator, and $\mathbf{U}_{0}$ is the initial state. $\mathbf{P}$ is a collection of parameters with default value in this model. Let $M_{t}$ be the nonlinear propagator related to equation (1) from time 0 to $t$, then the solution at time $t$ can be written as:

$$
\mathbf{U}(t)=M_{t}\left(\mathbf{U}_{0}, \mathbf{P}\right) .
$$

The solution without perturbation (initial perturbation $\mathbf{u}_{0}$ or parameter perturbation $\mathbf{p}_{0}$ ) can be viewed as the reference state at time $t$. If we superimpose a parameter perturbation $\mathbf{p}_{0}$ on the initial parameter(s), the solution at time $t$ becomes $M_{t}\left(\mathbf{U}_{0}, \mathbf{P}+\mathbf{p}_{0}\right)$ and may have difference with the reference state. The difference is described as

$$
d(t)=M_{t}\left(\mathbf{U}_{0}, \mathbf{P}+\mathbf{p}_{0}\right)-M_{t}\left(\mathbf{U}_{0}, \mathbf{P}\right) .
$$

Since our aim is to find the parameter perturbation that has the largest gap with the reference state, the objective function of CNOP-P method represents as follows:

$$
\begin{aligned}
& J\left(\mathbf{p}_{0}^{*}\right)=\max _{\mathbf{p}_{0} \in \Omega} J\left(\mathbf{p}_{0}\right) . \\
& J\left(\mathbf{p}_{0}\right)=\|\mathrm{d}(t)\|=\left\|M_{t}\left(\mathbf{U}_{0}, \mathbf{P}+\mathbf{p}_{0}\right)-M_{t}\left(\mathbf{U}_{0}, \mathbf{P}\right)\right\|,
\end{aligned}
$$

where $\mathbf{p}_{0}^{*}$ is the optimal perturbation, and $J$ is the objective function of this extreme value problem. $\Omega$ is the feasible domain of the parameter perturbation and satisfies $\left\|\mathbf{p}_{0}\right\| \leq \beta \mathbf{P}, \mathbf{p}_{0} \varepsilon \Omega$. $\beta$ denotes the proportion of the default value and is set to $20 \%$ to ensure the rationality. In this work, the integral operator $M_{t}$ can be viewed as the quantization process of NAOI. To portray the NAO definitely, we choose a blocking indicator proposed by Liu [48] as the NAOI, which is defined as SLP anomaly projection on the NAO pattern:

$$
\mathrm{NAOI}=\frac{\left\langle\mathrm{SLP}_{\mathrm{NAO}}, \mathrm{SLP}_{a}\right\rangle}{\left\langle\mathrm{SLP}_{\mathrm{NAO}}, \mathrm{SLP}_{\mathrm{NAO}}\right\rangle},
$$

where $<>$ denotes the Euclidean inner product; SLP $_{\mathrm{NAO}}$ is the NAO anomaly pattern extracted by empirical orthogonal function (EOF), and the $\operatorname{SLP}_{a}$ is obtained by subtracting the climatological mean from the SLP output. Combining the equations (5) and (6), the objective function is described as

$$
\begin{aligned}
J\left(\mathbf{p}_{0}\right) & =|\Delta \mathrm{NAOI}|=\left|\mathrm{NAOI}_{\text {pert }}-\mathrm{NAOI}_{\text {refer }}\right| \\
& =\left|\frac{\left\langle M_{t}\left(\mathbf{U}_{0}, \mathbf{P}+\mathbf{p}_{0}\right)-M_{t}\left(\mathbf{U}_{0}, \mathbf{P}\right), \mathrm{SLP}_{\mathrm{NAO}}\right\rangle}{\left\langle\mathrm{SLP}_{\mathrm{NAO}}, \mathrm{SLP}_{\mathrm{NAO}}\right\rangle}\right| .
\end{aligned}
$$

Since the NAO has two types of phases, the positive $\left(\mathrm{NAO}^{+}\right)$and the negative $\left(\mathrm{NAO}^{-}\right)$, we focus on the maximum of the absolute value of the NAOI difference. To simplify the problem, the NAO events are considered to happen when $|\triangle \mathrm{NAOI}|>1$.

For searching CNOP-P, we apply two novel intelligence algorithms to deal with different scales of parameters: PSO and CMA-ES. These two algorithms break the limitation of traditional methods for solving CNOP and have a better performance. The architecture of the solving system is displayed in Figure 1.

2.3. PSO. At first, the parameters that are sensitive to NAO events need to be picked out from all model parameters using linear perturbation. Then the largest impact of each single-parameter and two-parameter combination is searched via the PSO algorithm. The PSO is a typical swarm intelligence algorithm for solving extreme optimization problems and is appropriate for solving CNOP.

The main idea of the PSO is to search for the position which has the largest fitness value with multiple particles [49]. Each iteration records the global optimal particle and approaches the optimal position by updating the particles' speed and position according to its optimal position and the optimal global position. Finally, the extreme value of the objective function and the parameter value are obtained with convergence to a stable state. For the $k^{\text {th }}$ iteration, the updating rules of the $i^{\text {th }}$ particle's speed and position are 


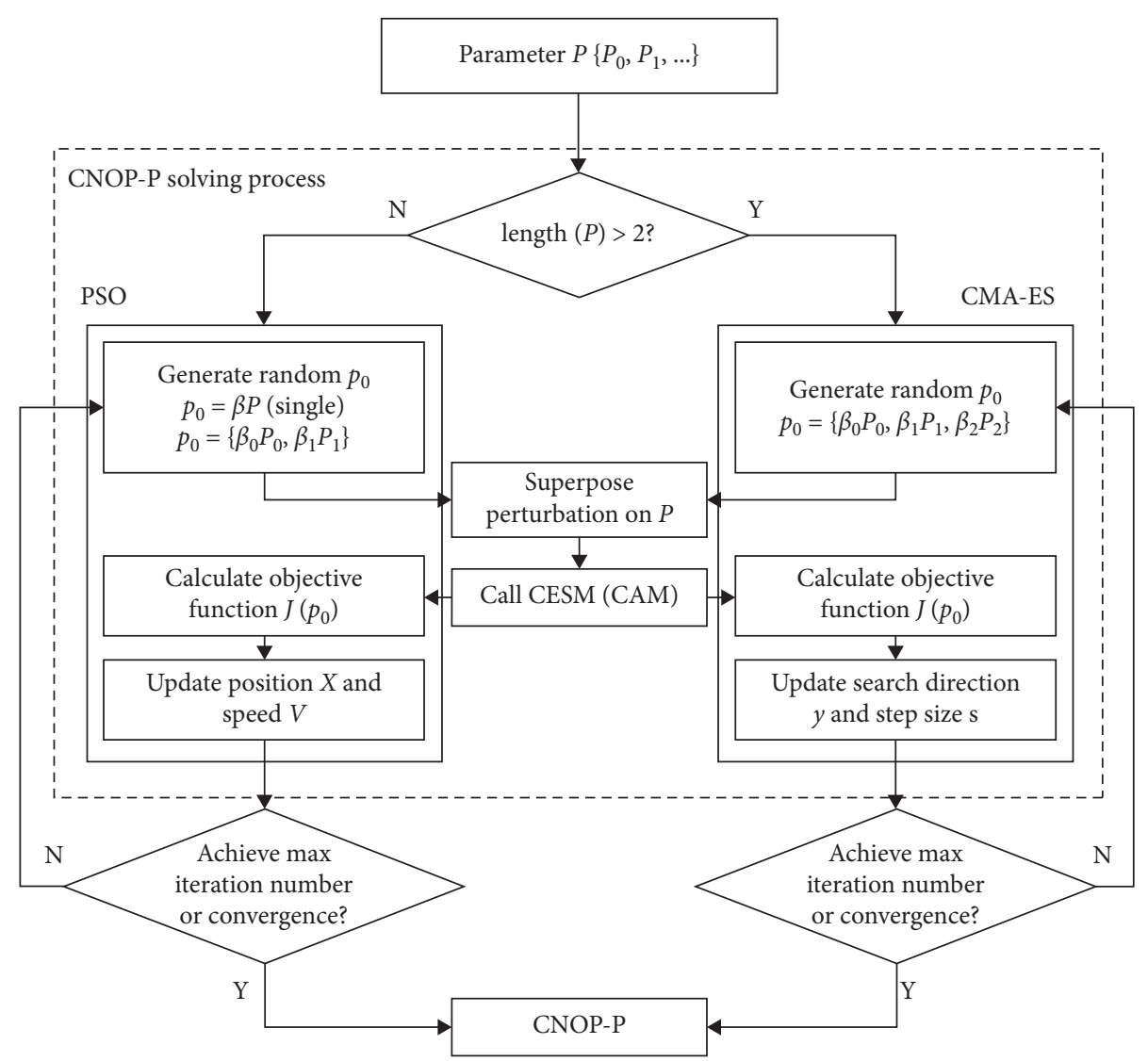

FIgURE 1: The architecture of the CNOP-P solving framework.

$$
\left\{\begin{array}{l}
\mathbf{V}_{i}^{k+1}=\mathbf{V}_{i}^{k}+c_{1} r_{1}\left(\mathbf{X}_{\mathrm{pb}}^{k}-\mathbf{X}_{i}^{k}\right)+c_{2} r_{2}\left(\mathbf{X}_{\mathrm{gb}}^{k}-\mathbf{X}_{i}^{k}\right), \\
\mathbf{X}_{i}^{k+1}=\mathbf{X}_{i}^{k}+\mathbf{V}_{i}^{k+1},
\end{array}\right.
$$

where $c_{1}$ is the self-awareness coefficient for historical selfoptimal position, and $c_{2}$ is the social-awareness coefficient for global optimal position of all particles. The empirical value of $c_{1}$ and $c_{2}$ are both set to 2. $r_{1}$ and $r_{2}$ are random values with uniform distribution in $[0,1] . \mathbf{X}_{\mathrm{pb}}^{k}$ denotes the self-optimal position of the current particle, and $\mathbf{X}_{\mathrm{gb}}^{k}$ denotes the global optimal position in the $k^{\text {th }}$ iteration.

2.4. CMA-ES. For multiparameter combination (parameter number $\geq 3$ ), we adopt CAM-ES, which is widely used for hyper-parameter adjustment in the field of machine learning, to investigate the most significant impact caused by multiple model parameters. CAM-ES is appropriate for complex optimization problems with a medium-scale number of parameters.

The core concept of the CMA-ES is to adjust the covariance matrix $\mathbf{C}$ in the normal distribution $N\left(\mathbf{m}_{t}, \boldsymbol{\sigma}_{t}^{2} \mathbf{C}_{t}\right)$ for updating the dependence between variables. The CMAES is performed in three main steps: (1) generate a new solution with sampling; (2) calculate the fitness value; (3) update the distribution parameters.
In each iteration, $\lambda$ solutions $(\lambda=x \log n, x \in N)$ are generated from $N\left(\mathbf{m}_{t}, \boldsymbol{\sigma}_{t}^{2} \mathbf{C}_{t}\right)$ :

$$
\mathbf{x}_{i}=\mathbf{m}_{t}+\boldsymbol{\sigma}_{t} \mathbf{y}_{i}, \quad \mathbf{y}_{i} \sim N(0, I),
$$

where $\mathbf{y}_{i}$ refers to the $i^{\text {th }}$ search direction and can be obtained from the eigen decomposition of the covariance matrix $\mathbf{C}_{t}=\mathbf{B D}_{\mathbf{z}_{i}}, \mathbf{z}_{i} \sim N(0, I)$ :

$$
\mathbf{y}_{i}=\mathbf{B D}_{\mathbf{z}_{i}}, \quad \mathbf{z}_{i} \sim N(0, I) .
$$

Then the fitness values of these solutions are calculated and are sorted in a descending (ascending) order for $\mathrm{NAO}^{+}$ $\left(\mathrm{NAO}^{-}\right)$. After that, the distribution parameters $\mathbf{m}_{t}$ and $\mathbf{C}_{t}$ are updated independently by a maximum likelihood estimate:

$$
\mathbf{m}_{t+1}=\sum_{i=1}^{\mu} \omega_{i} \mathbf{x}_{i: \lambda}=\mathbf{m}_{t}+\sum_{i=1}^{\mu} \omega_{i}\left(\mathbf{x}_{i \cdot \lambda}-\mathbf{m}_{t}\right),
$$

where $\omega_{i}$ satisfies $\omega_{1} \geq \omega_{i} \geq \omega_{\mu}>0$ to highlight the solution with larger fitness value.

The update of the covariance matrix $\mathbf{C}$ contains two parts: the evolution path and the natural gradient. The construction mode of the evolution path can be displayed as follows:

$$
\mathbf{p}_{t+1}=(1-c) \mathbf{p}_{t}+\sqrt{c(2-c)} \sqrt{\mu_{\omega}} \frac{\mathbf{m}_{t+1}-\mathbf{m}_{t}}{\mathbf{\sigma}_{t}}
$$


where $\mu_{\omega}$ is set to $1 / \sum_{i=1}^{\mu} \omega_{i}^{2}$ since $\sqrt{\mu_{\omega}}\left(\left(\mathbf{m}_{t+1}-\mathbf{m}_{t}\right) / \sigma_{t}\right)=$ $\sqrt{\mu_{\omega}} \sum_{i=1}^{\mu} \omega_{i} \mathbf{y}_{i: \lambda} \sim N\left(0, \mathbf{C}_{t}\right)$. c denotes the learning rate, and the factor $\sqrt{c(2-c)}$ obeys the stationary condition $(1-c)^{2}+(\sqrt{c(2-c)})^{2}=1$, which make $\mathbf{p}_{t+1} \sim N\left(0, \mathbf{C}_{t}\right)$.

Combining with the formula (12), the update of the covariance matrix $\mathbf{C}$ can be written as

$$
\begin{aligned}
\mathbf{C}_{t+1}= & \left(1-c_{1}-c_{\mu}\right) \mathbf{C}_{t}+c_{1} \mathbf{p}_{t+1} \mathbf{p}_{t+1}^{T} \\
& +c_{\mu} \sum_{i=1}^{\mu} \omega_{i}\left(\frac{\mathbf{x}_{i: \lambda}-\mathbf{m}_{t}}{\mathbf{\sigma}_{t}}\right)\left(\frac{\mathbf{x}_{i: \lambda}-\mathbf{m}_{t}}{\mathbf{\sigma}_{t}}\right)^{T},
\end{aligned}
$$

where the second item is to treat the evolution path as the search direction, and the third item is to evolve according to the natural gradient of the $\mathbf{C}$. The learning rate $c_{1}$ and $c_{\mu}$ are inversely proportional to the number of parameters; that is, $c_{1} \approx\left(2 / n^{2}\right), c_{\mu} \approx\left(\mu_{\omega} / n^{2}\right)$.

The adjust method used in CMA-ES is cumulative step size adaptation (CSA), which adjusts step size according to the angle between consecutive search directions. In other words, the consecutive search directions should be conjugated. The conjugate evolution path is

$$
\begin{aligned}
& \mathbf{s}_{t+1}=\left(1-c_{\sigma}\right) \mathbf{s}_{t}+\sqrt{c_{\sigma}\left(2-c_{\sigma}\right)} \sqrt{\mu_{\omega}} \mathbf{C}^{-(1 / 2)} \frac{\mathbf{m}_{t+1}-\mathbf{m}_{t}}{\boldsymbol{\sigma}_{t}}, \\
& \boldsymbol{\sigma}_{t+1}=\boldsymbol{\sigma}_{t} e^{\left(c_{\sigma} / d_{\sigma}\right)\left(\left(s_{t+1} / E N(0, I)\right)-1\right)\left(s_{t+1} / E N(0, I)\right)},
\end{aligned}
$$

where $\mathbf{s}_{t+1} \sim N(0, I)$ is in steady condition. Thus, $\left\|s_{t+1}\right\| \sim \chi(n)$, and $E\left(\left\|\boldsymbol{s}_{t+1}\right\|\right)=\sqrt{n}$. Since $E\left(\left\|\mathbf{s}_{t+1}\right\|\right)=\sqrt{n}$, it proves the intuitive idea of the CSA. $\mathbf{c}_{\sigma}$ and $\mathbf{d}_{\sigma}$ are the coefficients of step size rangeability. In general, $c_{\sigma} \propto(1 / n)$, $d_{\sigma}>1$.

\section{Experimental Settings}

CESM 1.2.2, along with CAM5.3, are adopted for NAO simulation during winter (DJF). We select $F$ as the component set and conduct the experiments on a daily average. $F$ integrates the atmospheric component, the land component, and the sea-ice component. The grids have 26 layers in the vertical under a resolution of $0.9^{\circ} \times 1.25^{\circ}$. The region we mainly focus is located in the North Atlantic area between $20^{\circ} \mathrm{N}$ and $80^{\circ} \mathrm{N}$ and between $90^{\circ} \mathrm{W}$ and $40^{\circ} \mathrm{E}$ with $65 \times 105$ grids, as shown in Figure 2.

In order to obtain a more comprehensive view of the model parameters, we perform the preliminary screening from all 237 parameters in these components. 48 available parameters with numeric type are sifted through their types and definitions. These parameters distribute in three components, atmosphere, ice, and river, and most of them are contained in the atmosphere component. In order to select sensitive parameters among them, we superpose the linear perturbations on the basic state for each parameter. The values of the perturbations are set from $1 \%$ to $20 \%$ of the default values, and the step size is $1 \%$. The parameters that would cause the variation of the NAOI can be regarded as the sensitive parameters. After the above steps, we obtain 28 sensitive parameters, which are displayed in Table 1.
Thereinto, p1-p25 are parameters in the atmosphere component, and p26-p28 are in the ice component. Then these sensitive parameters are analyzed with further selection and combination in subsequent sections. First, we calculate $\triangle \mathrm{NAOI}$ by varying selected parameters on a oneby-one basis, then each parameter is searched by PSO with 30 particles and 50 iterations. After that, the top $10 \mathrm{pa}-$ rameters for $\mathrm{NAO}^{+}$and $\mathrm{NAO}^{-}$are, respectively, combined into pairs, and $2 * C_{10}^{2}=90$ groups of parameter combinations are built. In this step, the CNOP-Ps are also solved by PSO, and the length of the parameter vector is 2. Finally, we select 5 most sensitive parameters (combinations) from 90 groups, and parameters are combined into triple-parameter subsets with $2 * C_{5}^{3}=20$ groups. The sensitivities of triple-parameter groups are explored by the CMA-ES method. A total of 166 CNOP-Ps with different scales are optimized in light of their fitness values.

Thereinto, p1-p5, p9-p10, and p22 are parameters directly related to physical variables and have specific ranges. Through consulting the historical data, the scopes of these parameters are listed in Table 2.

The experiments are conducted on the Tianhe- 2 supercomputer, with two Xeon E5 12-core CPUs and three Xeon Phi 57-core coprocessors for each node.

\section{Results and Analysis}

4.1. The Maximum Uncertainties due to Parameter Perturbations for Two Phases of NAO. The maximal extent of uncertainty is quantified via $\triangle \mathrm{NAOI}$ in equation (7). $\triangle \mathrm{NAOI}$ describes the NAOI difference compared with the reference state. The reference state can be obtained by running CESM with default parameter values. The greatest variation of positive $\triangle \mathrm{NAOI}$ and negative $\triangle \mathrm{NAOI}$ triggered by single parameters is illustrated in Figures 3 and 4. The range of $\triangle \mathrm{NAOI}^{+}$is from 0.22 to 1.45 , and p10 (Minimum relative humidity for low stable clouds) achieves the largest difference, while $\mathrm{p} 1\left(\mathrm{CH}_{4}\right.$ volume mixing ratio) has the least influence on the $\mathrm{NAO}^{+}$. In Figure $4, \Delta \mathrm{NAOI}^{-}$ranges from -0.54 to -1.70 . The sensitivity extent of each parameter is approximately similar to the $\mathrm{NAO}^{+} \cdot \mathrm{p} 10$ (minimum relative humidity for low stable clouds) is still the leading parameter in the aspect of sensitivity, and p22 (total solar irradiance) becomes the most relevant factor. According to our definition in Section 2, NAO events are considered to occur when $|\triangle \mathrm{NAOI}|>1$. As shown in Figure 4, some of the parameters can lead the $\mathrm{NAO}^{-}$events to happen, and the maximum value of $|\triangle \mathrm{NAOI}|$ is even greater than 1.5 (see $\mathrm{p} 22$ in Figure 4). Although not all the cases develop into NAO events, the uncertainties between prediction and observation owing to parameter perturbations are nonnegligible.

For further experiments, the top 10 parameters that achieve higher positive $\triangle \mathrm{NAOI}$ and lower negative $\triangle \mathrm{NAOI}$ are listed in Tables 3 and 4 . In these tables, parameter values at the maximum of the objective function are also reported.

To explore which parameter(s) have the highest sensitivities to NAO events more comprehensively, in addition to single parameters, parameter combinations need to be considered. The top 10 sensitive parameters (Tables 3 and 4) 


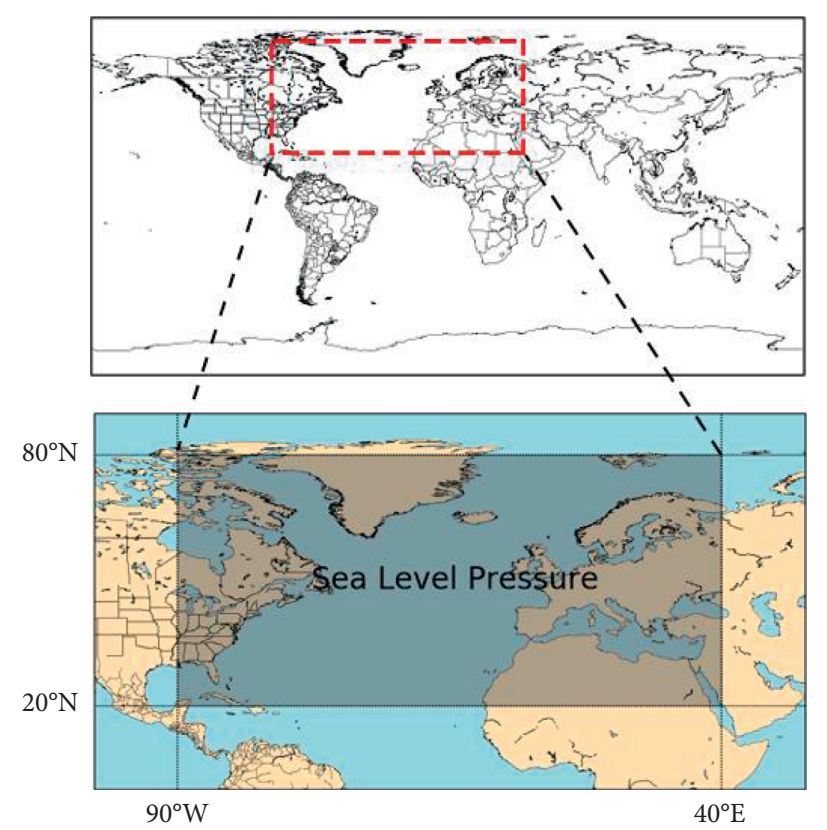

Figure 2: The region of NAO events.

TABLE 1: The 28 sensitive parameters screened by linear perturbations.

\begin{tabular}{|c|c|c|c|}
\hline Number & Parameter & Description & Default value \\
\hline p1 & ch4vmr & $\mathrm{CH}_{4}$ volume Mixing ratio & $1.76 * 10^{-6}$ \\
\hline p2 & $\mathrm{co} 2 \mathrm{vmr}$ & $\mathrm{CO}_{2}$ volume Mixing ratio & $3.67 * 10^{-4}$ \\
\hline p3 & fl1vmr & $\mathrm{CCl}_{3} \mathrm{~F}$ volume Mixing ratio & $6.53 * 10^{-10}$ \\
\hline p4 & $\mathrm{f} 12 \mathrm{vmr}$ & $\mathrm{CCl}_{2} \mathrm{~F}_{2}$ volume Mixing ratio & $5.35 * 10^{-10}$ \\
\hline p5 & $\mathrm{n} 2 \mathrm{ovmr}$ & $\mathrm{N}_{2} \mathrm{O}$ volume Mixing ratio & $3.16 * 10^{-7}$ \\
\hline p6 & cldfrc_dp1 & Parameter for deep convection cloud fraction & 0.1 \\
\hline p7 & cldfrc_dp2 & Parameter for deep convection cloud fraction & 500.0 \\
\hline p8 & cldfrc_premib & Bottom height for midlevel liquid stratus fraction & $7.5 * 10^{4}$ \\
\hline p9 & cldfrc_rhminh & Minimum relative humidity for high stable clouds & 0.77 \\
\hline p10 & cldfrc_rhminl & Minimum relative humidity for low stable clouds & 0.92 \\
\hline p11 & cldfrc_rhminl_adj_land & Adjustment to rhminl for land without snow cover & 0.1 \\
\hline p12 & cldfrc_sh 1 & Parameter for shallow convection cloud fraction & 0.04 \\
\hline p13 & cldfrc_sh2 & Parameter for shallow convection cloud fraction & 500.0 \\
\hline p14 & cldsed_ice_stokes_fac & Factor applied to the ice fall velocity & 1.0 \\
\hline p15 & cldwat_conke & Tunable constant for evaporation of precip & $5.0 * 10^{-6}$ \\
\hline p16 & cldwat_icritc & Threshold for autoconversion of cold ice & $1.8 * 10^{-5}$ \\
\hline p17 & cldwat_icritw & Threshold for autoconversion of warm ice & $2.0 * 10^{-4}$ \\
\hline p18 & cldwat_r3lcrit & Critical radius at which autoconversion become efficient & $1.0 * 10^{-5}$ \\
\hline p19 & fcrit2 & Critical froude number squared & 1.0 \\
\hline p20 & hkconv_c0 & Rain water autoconversion coefficient & $1.0 * 10^{-4}$ \\
\hline p21 & hkconv_cmftau & Characteristic adjustment time scale & $1.8 * 10^{3}$ \\
\hline p22 & solar_const & Total solar irradiance $\left(\mathrm{W} / \mathrm{m}^{2}\right)$ & $1.36 * 10^{3}$ \\
\hline p23 & zmconv_c0_lnd & Autoconversion coefficient over land & $3.5 * 10^{-3}$ \\
\hline p24 & zmconv_c0_ocn & Autoconversion coefficient over ocean & $3.5 * 10^{-3}$ \\
\hline p25 & zmconv_ke & Tunable evaporation efficiency & $1.0 * 10^{-6}$ \\
\hline p26 & dt_mlt_in & Melt onset temperature tunable parameter & 1.5 \\
\hline p27 & $\bar{r} \_s n w$ & Snow grain radius tunable parameter & 1.5 \\
\hline p28 & rsnw_melt_in & Maximum melting snow grain radius tunable parameter & $1.5 * 10^{3}$ \\
\hline
\end{tabular}

of $\mathrm{NAO}^{+}$and $\mathrm{NAO}^{-}$are combined in pairs, and totaling 90 groups of two-parameter combinations are constructed. We implement the sensitivity experiments on these combinations and compare them with single parameters. PSO is still employed to find the extremum of the objective function, with 30 particles and 50 iteration steps for each combination. The parameter perturbation is organized into the vector with a size of 2 and is assigned as the position of particles. We plot heatmaps to visually display distributions of the largest (lowest) $\triangle$ NAOI due to these combinations. Figure 5 shows 
the results of 45 parameter sets related to the $\mathrm{NAO}^{+}$, and the diagonal refers to the largest uncertainties corresponding to single parameters. For instance, the first block at the upper left denotes the $\triangle$ NAOI triggered by $\mathrm{p} 6$ only. Values on the diagonal are smaller than that of the same row or the same column, indicating a more significant effect of parameter combinations. In Figure 5, we can see some grids with darker colors obviously, such as p10 (Minimum relative humidity for low stable clouds) and p20 (Rain water autoconversion coefficient), p10 and p15 (Constant for evaporation of precip), and p10 and p22 (Total solar irradiance). The areas with darker colors are also concentrated in the combination of $\mathrm{p} 9$ and p10 with other parameters. Conversely, combinations with p7 (parameter for deep convection cloud fraction 2) or p21 (characteristic adjustment time scale) are in lighter colors with lower sensitivities.

Figure 6 depicts the results of two-parameter combinations for $\mathrm{NAO}^{-}$. Similarly, the ranks of sensitivity for single parameters is relatively lower. However, there are exceptions, such as the effect of $\mathrm{p} 7$ (parameter for deep convection cloud fraction 2) and p20 (rain water autoconversion coefficient) combination is inferior to that of $\mathrm{p} 7$ or p20, and the same goes for p6 (parameter for deep convection cloud fraction 1) and p7. A cross-dark ribbon appears in Figure 6, and it demonstrates that the parameter combinations containing $\mathrm{p} 15$ (Constant for evaporation of precip) or $\mathrm{p} 22$ (Total solar irradiance) are highly sensitive to the $\mathrm{NAO}^{-}$. Compared to the other nine parameters, p28 (Maximum melting snow grain radius tunable parameter) has less contribution to the form of the $\mathrm{NAO}^{-}$events.

In the next group of experiments, 10 parameters ( 5 for $\mathrm{NAO}^{+}$, and the other 5 for $\mathrm{NAO}^{-}$) would be determined according to the results of two-parameter combinations, and relative insensitive parameters are eliminated. Then they would be grouped into combinations with three parameters, for a total of 20 sets. At first, we list the top 10 parameter combinations with the largest $\triangle \mathrm{NAOI}$ value in Figure 5, sequentially recorded as $\mathrm{cl}$ to $\mathrm{c} 10$. It can be seen that $\mathrm{p} 10$ (minimum relative humidity for low stable clouds) occurs most often in Table 5. What's more, p10 is also the single parameter most closely related to the $\mathrm{NAOI}^{+}$. p9 (minimum relative humidity for high stable clouds) is another critical parameter that can promote the occurrence of the $\mathrm{NAO}^{+}$. The complex association has been detected between relative humidity and the NAO in winter (DJF) [50], and the relative humidity can also be affected by the NAO forcing [51]. p9 and $\mathrm{p} 10$ are selected for the following experiments, and other parameters (p6, p15, and p22) are picked by balancing the fitness value and the average ranking of $\triangle \mathrm{NAOI}$. In Table 5 , the $\triangle$ NAOI are all greater than 1 and are significantly higher than the single-parameter results in Table 3 . The top 10 parameter combinations for $\mathrm{NAO}^{-}$named $\mathrm{c} 11$ to $\mathrm{c} 20$ are presented in Table 6, and parameters (p15, p20, p21, p22, and p24) are chosen in the same way. The high sensitivities of p22 (Total solar irradiance) are demonstrated for most sets. With decreasing solar activity, the increasing pressure difference between Azores low and Iceland high is observed. The values of $\triangle \mathrm{NAOI}$ are further reduced compared to Table 4 , and most of them are less than -2 . We can see that a large margin
TABLE 2: The ranges of some parameters according to historical data.

\begin{tabular}{lccc}
\hline Number & Parameter & Parameter range & Unit \\
\hline p1 & ch4vmr & $1.70-1.90$ & $\mathrm{ppm}$ \\
p2 & co2vmr & $370-430$ & $\mathrm{ppm}$ \\
p3 & f11vmr & $260-500$ & $\mathrm{ppt}$ \\
p4 & f12vmr & $530-830$ & $\mathrm{ppt}$ \\
p5 & n2ovmr & $311-328$ & $\mathrm{ppb}$ \\
p9 & cldfrc_rhminh & $33 \%-61 \%$ & - \\
p10 & cldfrc_rhminl & $33 \%-61 \%$ & - \\
p22 & solar_const & $1.356 * 10^{3}-1.364 * 10^{3}$ & $\mathrm{~W} / \mathrm{m}^{2}$ \\
\hline
\end{tabular}

of error can be caused due to deviations of several parameters. It is entirely possible that the qualitative analysis of the prediction would make a huge difference because of parameter errors.

The experiments with three-parameter combinations are performed with parameters that appear more frequently in Tables 5 and 6 . In settings of CMA-ES, the initial solution is set to the vector containing default values of the three parameters, and the initial standard deviation is set to 0.5. These combinations are sorted in descending order (ascending order for $\mathrm{NAO}^{-}$) by fitness values ( $\triangle \mathrm{NAOI}$ ), marking them as c21 to c40. The results of the two phases are illustrated in Figures 7 and 8 with parallel coordinate systems. The red dot stands for the default value, and the fitness value displays in the last coordinate axis. Taking c30 in Figure 7 as an example, the $\mathrm{c} 30$ represented by the red line is composed of $\mathrm{p} 6, \mathrm{p} 15$, and $\mathrm{p} 22$ (p9 and p10 are set as the default). When the fitness value achieves a maximum of 1.43 , the values of these three parameters are $0.102,4.98 * 10^{-6}$, and $1.36206 * 10^{3}$, respectively. Other combinations are processed in the same manner, and the details are reported in Table 7. The multiple factors mutually promote the process of the NAO, and the final state is not equivalent to a simple superposition of the effects of each parameter acting alone. $\triangle \mathrm{NAOI}$ values are generally larger (smaller) than $1.5(-1.5)$. In addition, a large gap is between these fitness values, especially for the $\mathrm{NAO}^{-}$, which is distributed between -1.5 and -3 (Table 8).

\subsection{The Evolution of NAOI with Parameter Perturbations.} In this section, we aim to trace changing process of the NAOI triggered by parameter perturbations during the prediction time, and the variation trend of the NAOI is illustrated to observe the evolution of the NAO (see Figure 9). The reference state is displayed with a red dotted line, and the perturbation states corresponding to the ten parameters mentioned in Tables 3 and 4 are shown as lines with different colors. The reference state denotes the final state without superposing perturbations. In Figure 9, different parameters appear different directions of variation for NAOI and fluctuate before converge to a stable state. In addition, the perturbation states and the reference state almost coincide in the first half of the prediction time. The nonlinear process takes effect in the final duration and develops into a diverse result.

Figure 10 shows the evolution of NAOI with two-parameter combinations for the two phases during the 


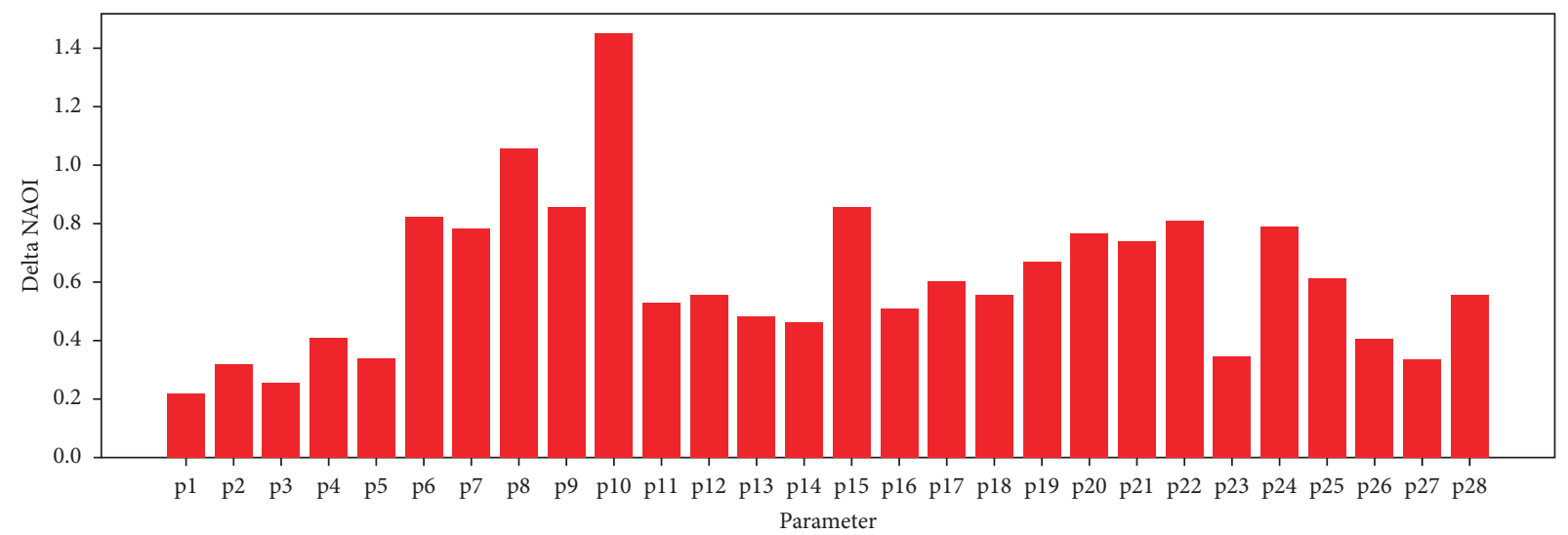

Figure 3: The largest positive $\Delta$ NAOI triggered by 28 parameters.

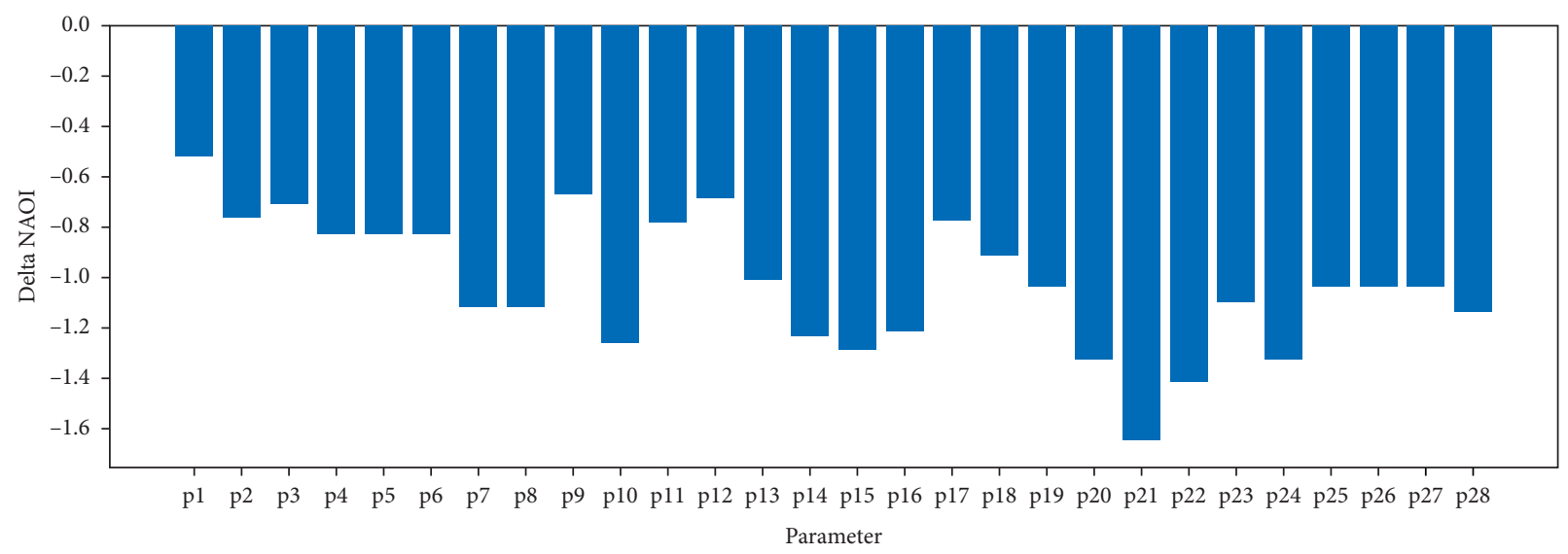

Figure 4: Similar to Figure 3, but for negative $\triangle$ NAOI.

TABLE 3: The top 10 parameters that cause the largest uncertainty for $\mathrm{NAO}^{+}$.

\begin{tabular}{lcc}
\hline Number & $\Delta$ NAOI $\left(\mathrm{NAO}^{+}\right)$ & Parameter value \\
\hline p10 & 1.45 & 0.61 \\
p8 & 1.06 & $8.81 * 10^{4}$ \\
p15 & 0.86 & $4.0 * 10^{-6}$ \\
p9 & 0.85 & 0.55 \\
p6 & 0.82 & 0.12 \\
p22 & 0.81 & $1.36194 * 10^{3}$ \\
p7 & 0.79 & 591.53 \\
p24 & 0.78 & $2.84 * 10^{-3}$ \\
p20 & 0.76 & $8.27 * 10^{-5}$ \\
p21 & 0.74 & $1.85 * 10^{3}$ \\
\hline
\end{tabular}

prediction time. The similarity with Figure 9 is that the fluctuation of the NAOI of each perturbation in the first-half phase is almost identical to the reference state. The trends of perturbations are diverse from each other in the last stage. Several perturbations tend to decrease in the later stage in spite of the higher NAOI. In the left chart of Figure 10, the perturbation flow remains on an upward trend. For $\mathrm{NAO}^{-}$, the perturbation flows of Figures 9 and 10 are basically in a state of continuous decline, and in Figure 10, the direction of
TABLE 4: Similar to Table 2, but for $\mathrm{NAO}^{-}$.

\begin{tabular}{lcc}
\hline Number & $\Delta \mathrm{NAOI}\left(\mathrm{NAO}^{-}\right)$ & Parameter value \\
\hline p21 & -1.70 & $1.99 * 10^{3}$ \\
p22 & -1.49 & $1.36230 * 10^{3}$ \\
p20 & -1.37 & $1.20 * 10^{-4}$ \\
p24 & -1.35 & $3.86 * 10^{-3}$ \\
p15 & -1.32 & $5.54 * 10^{-6}$ \\
p10 & -1.30 & 0.58 \\
p14 & -1.27 & 1.20 \\
p16 & -1.25 & $1.72 * 10^{-5}$ \\
p28 & -1.17 & $1.64 * 10^{3}$ \\
p7 & -1.15 & 574.87 \\
\hline
\end{tabular}

the NAOI variation in the negative phase is more consistent than that of Figure 9.

Figure 11 displays the variation of the three-parameter combinations for NAOI value, which has a slight enhancement than the two-parameter combinations.

4.3. The SLP Anomaly Patterns. In general, NAO events occur with the abnormal high (low) NAOI as well as the dipole mode of anomaly SLP, and the spatial distribution of 


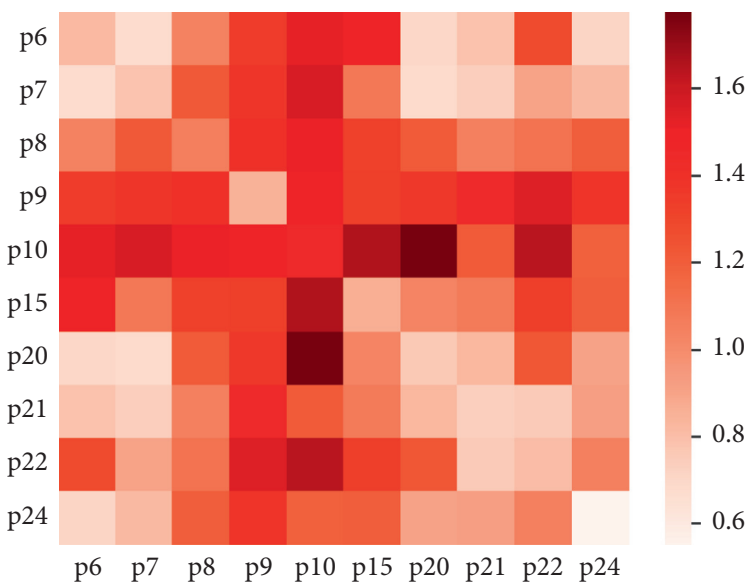

FIgURE 5: $\triangle \mathrm{NAOI}\left(\mathrm{NAO}^{+}\right)$for two-parameter combinations with parameters in Table 3.

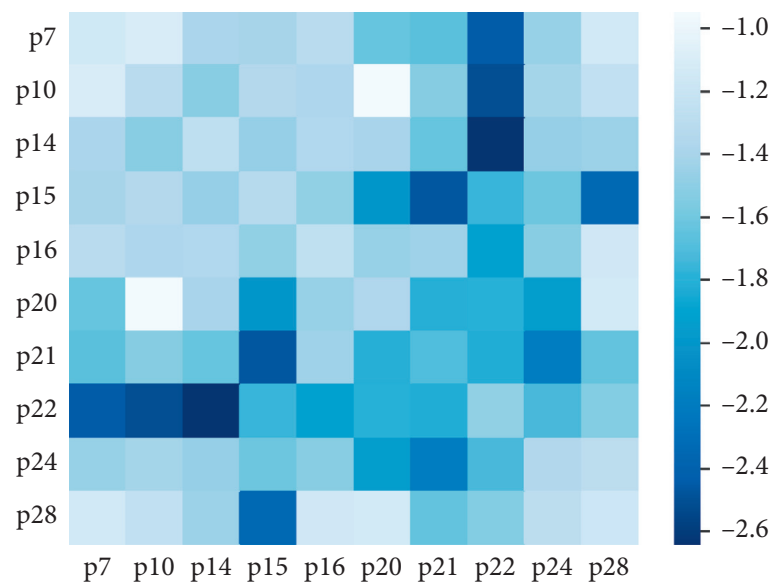

Figure 6: $\triangle \mathrm{NAOI}\left(\mathrm{NAO}^{-}\right)$for two-parameter combinations with parameters in Table 4.

SLP reflects the variations of the NAO. To investigate the impact of parameter perturbations, the responses of the SLP field to model parameters are visualized in Figures 12-17. The SLP field is presented in the form of the difference between perturbation state and reference state, and the critical region is selected as the area of North Atlantic Ocean. As it can be seen in Figure 12, although all of them have a relative high NAOI, the strong positive center and negative center only appear in patterns of $\mathrm{p} 9, \mathrm{p} 10$, and $\mathrm{p} 22$. The pressure structures of $\mathrm{p} 9, \mathrm{p} 10$, and $\mathrm{p} 22$ show a northeastsouthwest tilt, with discontinuous high-pressure areas and messy pressure cores. In spite of the opposite pressure fields in the both sides of $60^{\circ} \mathrm{N}$, no obvious pressure difference is generated in the rest of subplots. It is indicated that the perturbation state of most single parameters cannot evolve into $\mathrm{NAO}^{+}$events. As for the mode of $\mathrm{NAO}^{-}$(see Figure 13), the patterns of $\mathrm{p} 21, \mathrm{p} 22, \mathrm{p} 15$, and $\mathrm{p} 16$ show the significant SLP difference with a range of $-3500 \mathrm{~Pa}$ to $4500 \mathrm{~Pa}$. The positive and negative pressure regions are respectively on either side of middle latitude, and a positive SLP anomaly occupies the south of the Greenland and Western Europe in these cases, resulting in the $\triangle \mathrm{NAOI}$ less than -1 . The westpositive patterns are formed with multiple pressure centers
TABLE 5: The top 10 two-parameter combinations that cause the largest uncertainty for $\mathrm{NAO}^{+}$.

\begin{tabular}{lccc}
\hline Number & Parameter & $\Delta$ NAOI $\left(\mathrm{NAO}^{+}\right)$ & Parameter value \\
\hline c1 & p10, p20 & 1.78 & $0.38,1.03 * 10^{-4}$ \\
c2 & p10, p15 & 1.66 & $0.51,4.78 * 10^{-6}$ \\
c3 & p10, p22 & 1.64 & $0.43,1.36222 * 10^{3}$ \\
c4 & p9, p24 & 1.62 & $0.53,3.67 * 10^{-3}$ \\
c5 & p7, p10 & 1.56 & $0.77,0.61$ \\
c6 & p9, p22 & 1.55 & $0.35,1.35875 * 10^{3}$ \\
c7 & p6, p10 & 1.52 & $0.097,0.56$ \\
c8 & p8, p10 & 1.51 & $7.25 * 10^{4}, 0.55$ \\
c9 & p9, p10 & 1.50 & $0.54,0.41$ \\
c10 & p6, p15 & 1.48 & $0.103,4.85 * 10^{-6}$ \\
\hline
\end{tabular}

scattered around the $50^{\circ} \mathrm{N}$. For most cases of single parameter, the SLP field is irregular and unstable or even no substantial difference. Specifically, p22 (Total solar irradiance) can exert marked influence on both $\mathrm{NAO}^{+}$and $\mathrm{NAO}^{-}$. It is concluded that the solar irradiance is the common factor related to different physical mechanisms of $\mathrm{NAO}^{+}$and $\mathrm{NAO}^{-}$.

Similar work was carried out for two-parameter combinations (see Figures 14 and 15), and SLP difference 
TABle 6: Similar to Table 5, but for $\mathrm{NAO}^{-}$.

\begin{tabular}{lccc}
\hline Number & Parameter & $\Delta$ NAOI $\left(\mathrm{NAO}^{-}\right)$ & Parameter value \\
\hline c11 & p14, p22 & -2.65 & $0.95,1.35650 * 10^{3}$ \\
c12 & p10, p22 & -2.50 & $0.48,1.36269 * 10^{3}$ \\
c13 & p15, p21 & -2.47 & $4.98 * 10^{-6}, 1.53 * 10^{3}$ \\
c14 & p7, p22 & -2.44 & $504.14,1.35886 * 10^{3}$ \\
c15 & p15, p28 & -2.34 & $4.90 * 10^{-6}, 1.53 * 10^{3}$ \\
c16 & p21, p24 & -2.18 & $1.99 * 10^{3}, 4.20 * 10^{-3}$ \\
c17 & p15, p20 & -2.00 & $9.78 * 10^{-5}, 4.77 * 10^{-6}$ \\
c18 & p20, p24 & -1.94 & $1.05 * 10^{-4}, 3.67 * 10^{-3}$ \\
c19 & p16, p22 & -1.92 & $1.72 * 10^{-5}, 1.36207 * 10^{3}$ \\
c20 & p21, p22 & -1.83 & $1.83 * 10^{3}, 1.35805 * 10^{3}$ \\
\hline
\end{tabular}

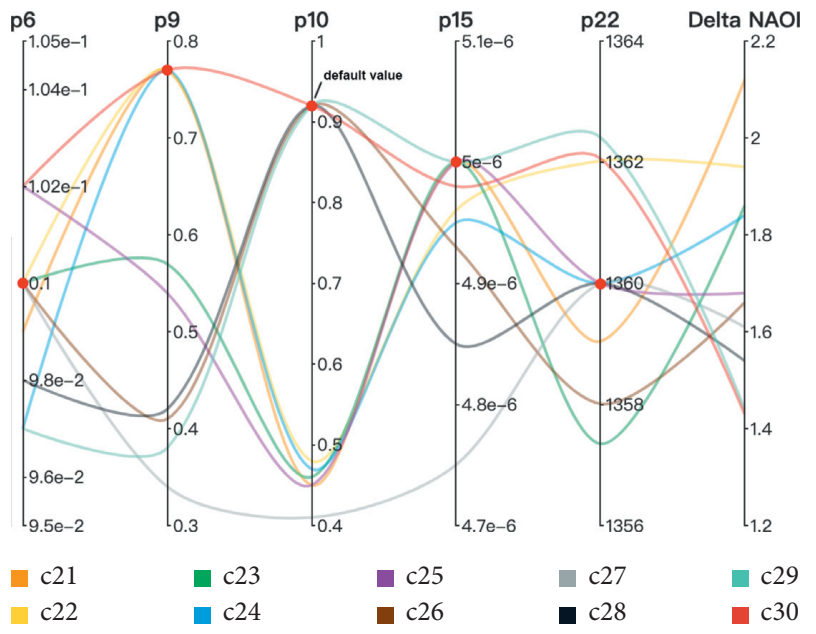

Figure 7: $\triangle \mathrm{NAOI}\left(\mathrm{NAO}^{+}\right)$of three-parameter combinations that are combined with 5 parameters selected from Table 5 . The red dot denotes the default value of the parameter.

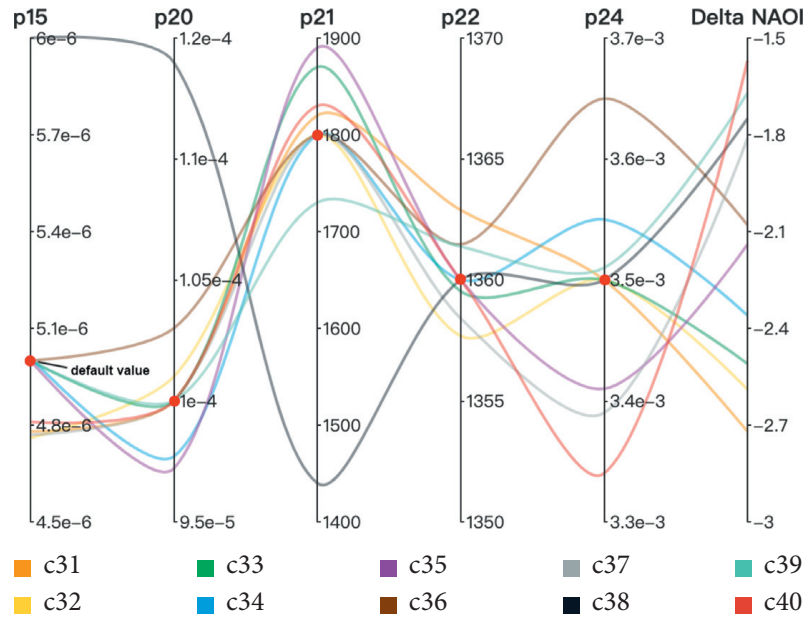

Figure 8: Similar to Figure 7, but for $\mathrm{NAO}^{-}$.

TABLE 7: The details of three-parameter combinations that cause the uncertainty in $\mathrm{NAO}^{+}$.

\begin{tabular}{lccc}
\hline Number & Parameter & $\Delta$ NAOI $\left(\mathrm{NAO}^{+}\right)$ & Parameter value \\
\hline c21 & p6, p10, p22 & 2.12 & $0.0997,0.45,1.35904 * 10^{3}$ \\
c22 & p10, p15, p22 & 1.94 & $0.48,4.92 * 10^{-6}, 1.36202 * 10^{3}$ \\
c23 & p9, p10, p22 & 1.86 & $0.57,0.46,1.35735 * 10^{3}$ \\
c24 & p6, p10, p15 & 1.84 & $0.097,0.47,4.95 * 10^{-6}$ \\
c25 & p6, p9, p10 & 1.68 & $0.102,0.54,0.45$ \\
c26 & p9, p15, p22 & 1.66 & $0.41,4.93 * 10^{-6}, 1.35801 * 10^{3}$ \\
c27 & p9, p10, p15 & 1.61 & $0.34,0.41,4.75 * 10^{-6}$ \\
c28 & p6, p9, p15 & 1.54 & $0.098,0.42,4.85 * 10^{-6}$ \\
c29 & p6, p9, p22 & 1.44 & $0.097,0.38,1.36241 * 10^{3}$ \\
c30 & p6, p15, p22 & 1.43 & $0.102,4.98 * 10^{-6}, 1.36206 * 10^{3}$ \\
\hline
\end{tabular}

TABle 8: The details of three-parameter combinations that cause the uncertainty in $\mathrm{NAO}^{-}$.

\begin{tabular}{llcc}
\hline Number & Parameter & $\Delta$ NAOI $\left(\mathrm{NAO}^{-}\right)$ & Parameter value \\
\hline c31 & P15, p21, p22 & -2.72 & $4.78 * 10^{-6}, 1.82 * 10^{3}, 1.36289 * 10^{3}$ \\
c32 & P15, p20, p22 & -2.59 & $4.76 * 10^{-6}, 1.01 * 10^{-4}, 1.35768 * 10^{3}$ \\
c33 & P20, p21, p22 & -2.51 & $1.00 * 10^{-4}, 1.87 * 10^{3}, 1.35953 * 10^{3}$ \\
c34 & P15, p20, p24 & -2.36 & $5.01 * 10^{-6}, 9.77 * 10^{-5}, 3.55 * 10^{-3}$ \\
c35 & p20, p21, p24 & -2.14 & $9.72 * 10^{-5}, 1.89 * 10^{3}, 3.41 * 10^{-3}$ \\
c36 & p20, p22, p24 & -2.08 & $1.03 * 10^{-4}, 1.36147 * 10^{3}, 3.65 * 10^{-3}$ \\
c37 & p15, p22, p24 & -1.81 & $4.77 * 10^{-6}, 1.35841 * 10^{3}, 3.39 * 10^{-3}$ \\
c38 & p15, p20, p21 & -1.75 & $6.00 * 10^{-6}, 1.14 * 10^{-4}, 1.44 * 10^{3}$ \\
c39 & p21, p22, p24 & -1.67 & $1.73 * 10^{3}, 1.36138 * 10^{3}, 3.51 * 10^{-3}$ \\
c40 & p15, p21, p24 & -1.57 & $4.81 * 10^{-6}, 1.83 * 10^{3}, 3.34 * 10^{-3}$ \\
\hline
\end{tabular}




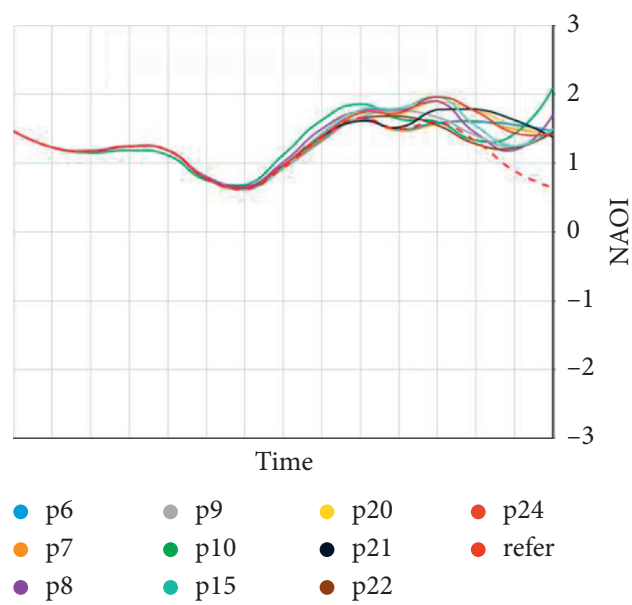

(a)

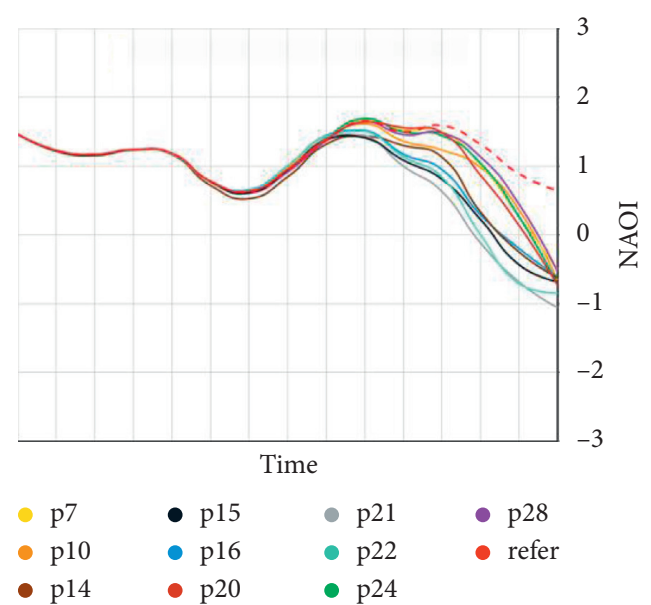

(b)

FIGURE 9: The NAOI variation flow of single parameters for two phases during the prediction time.
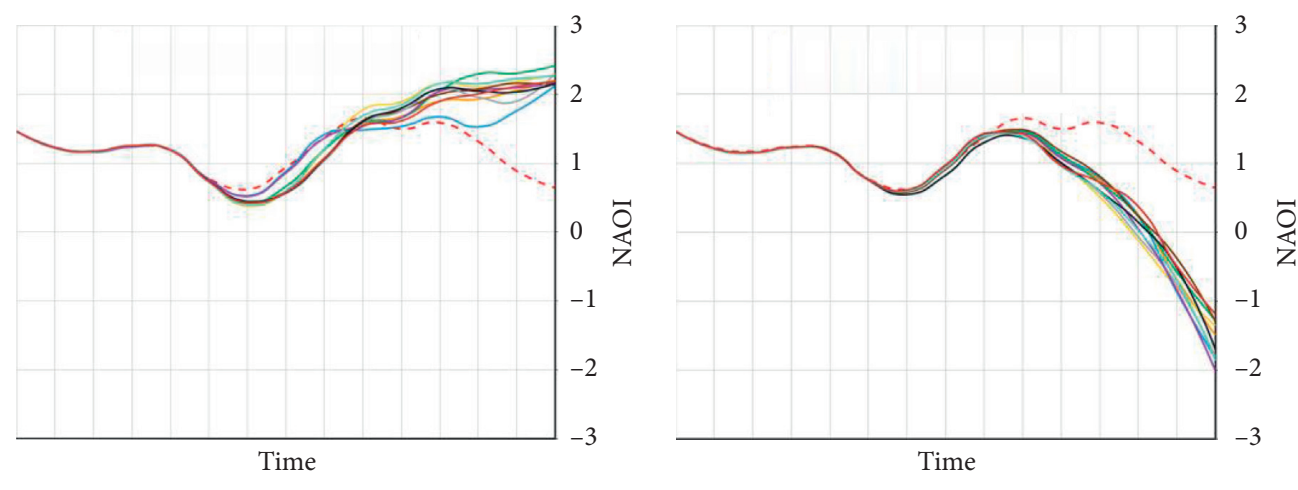

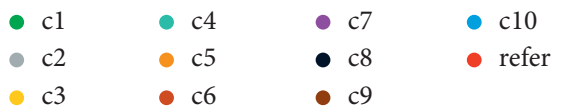

(a)

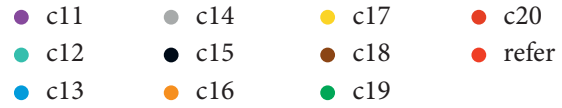

(b)

FIgURE 10: Similar to Figure 9, but for two-parameter combinations.

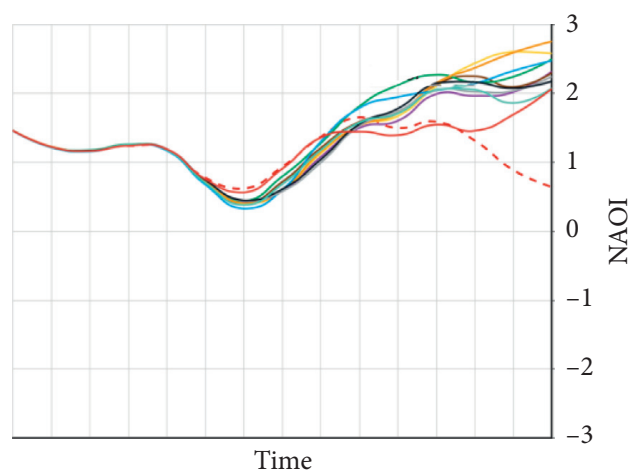

$\begin{array}{llll}-\mathrm{c} 21 & \bullet \mathrm{c} 24 & \bullet \mathrm{c} 27 & \bullet \mathrm{c} 30 \\ \mathrm{c} 22 & \bullet \mathrm{c} 25 & \bullet \mathrm{c} 28 & \bullet \mathrm{refer} \\ -\mathrm{c} 23 & \bullet \mathrm{c} 26 & \bullet \mathrm{c} 29 & \end{array}$

(a)

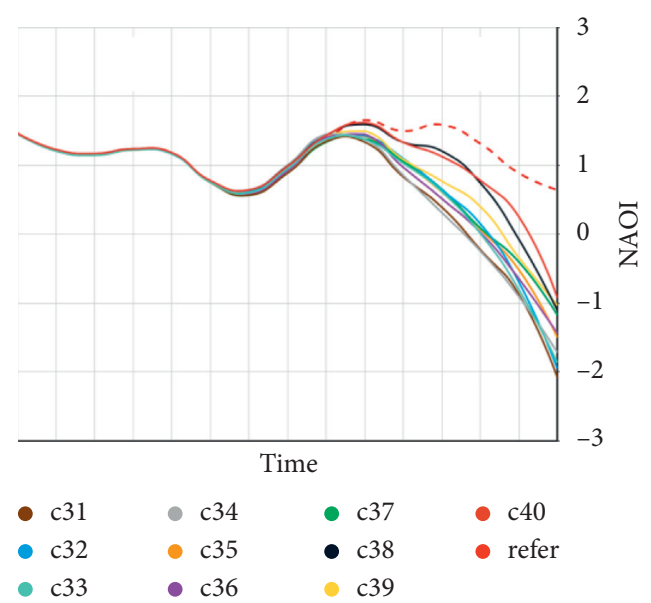

(b)

Figure 11: Similar to Figure 9, but for three-parameter combinations. 

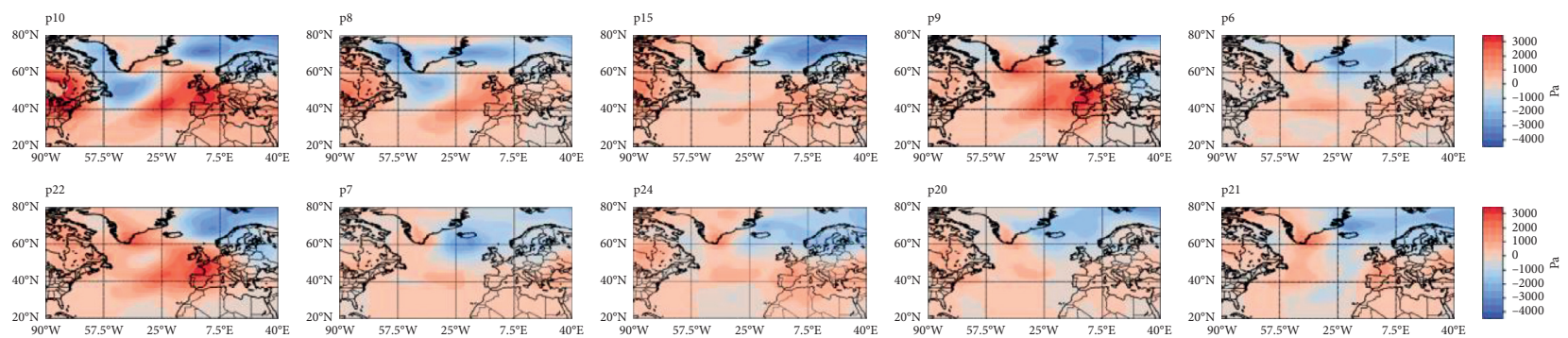

FIgURE 12: The SLP difference triggered by single parameters for $\mathrm{NAO}^{+}$.
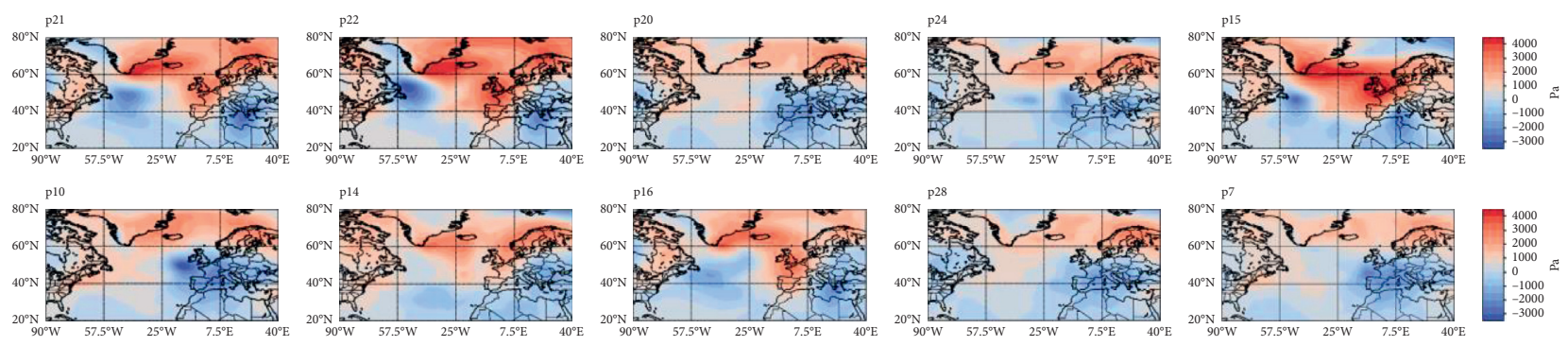

Figure 13: Similar to Figure 12, but for $\mathrm{NAO}^{-}$.
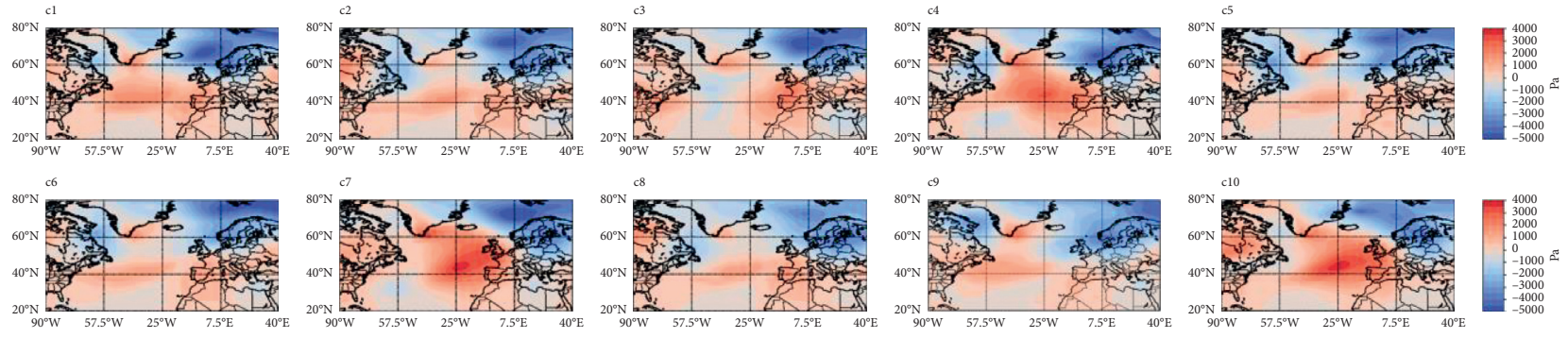

FIgURE 14: The SLP difference triggered by two-parameter combinations for $\mathrm{NAO}^{+}$.
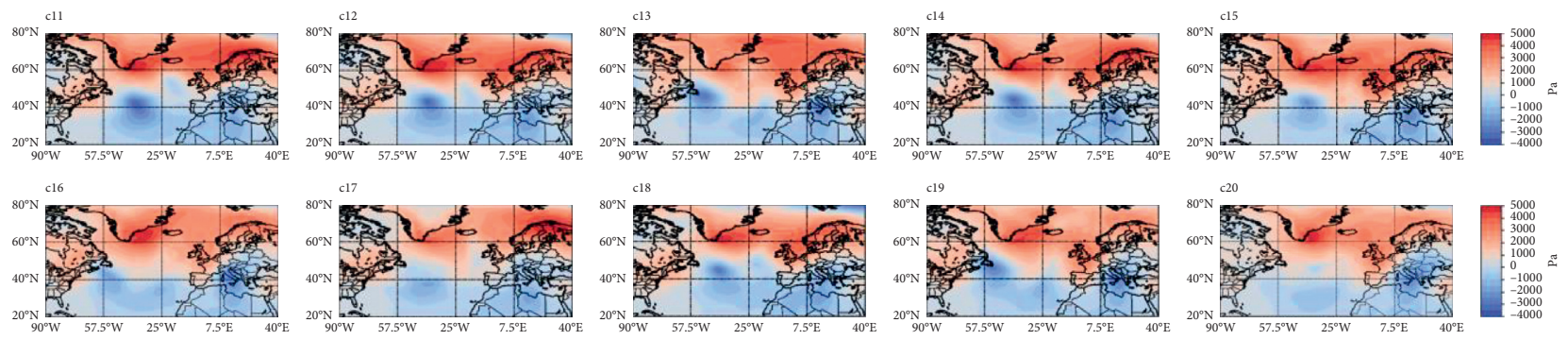

FIgURE 15: Similar to Figure 14, but for $\mathrm{NAO}^{-}$.
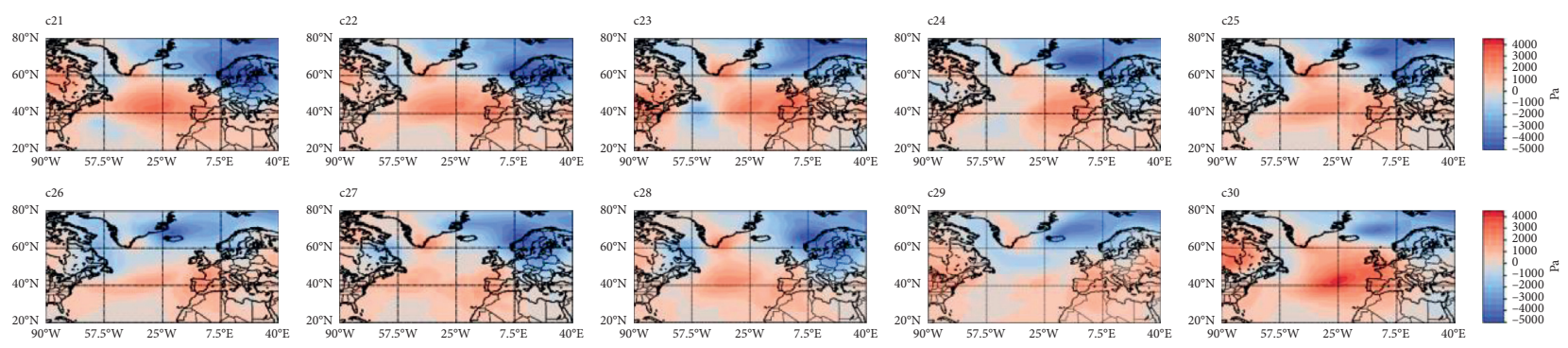

FIgURE 16: The SLP difference triggered by three-parameter combinations for $\mathrm{NAO}^{+}$. 

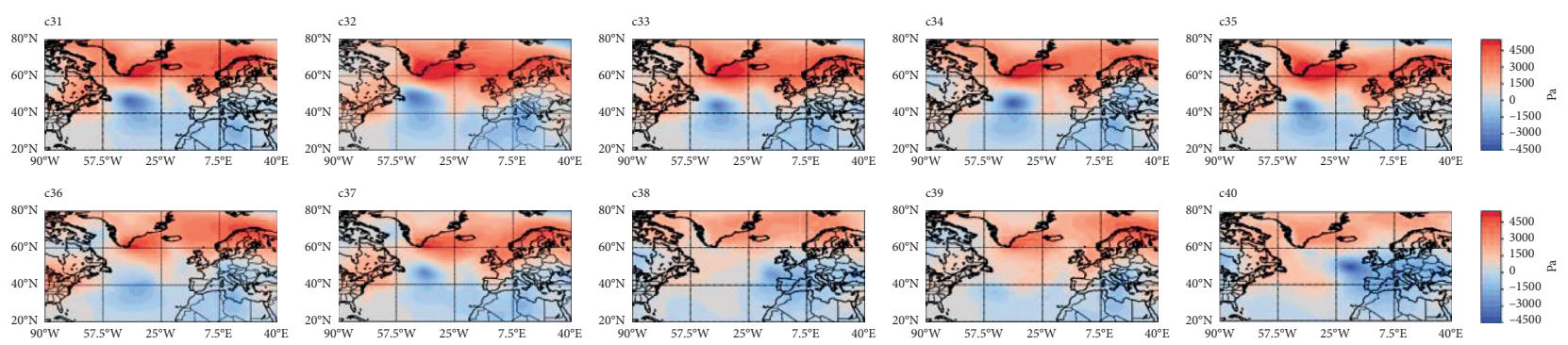

Figure 17: Similar to Figure 16, but for $\mathrm{NAO}^{-}$.

patterns for these combinations are plotted to investigate whether anomalous NAOI and typical meridional dipole occur simultaneously. The SLP difference is generally intensive than that of single parameters, and it ranges from $-5000(-4000)$ to $4000(5000) \mathrm{Pa}$ in $\mathrm{NAO}^{+}\left(\mathrm{NAO}^{-}\right)$. What's more, pressure cores are more prominent and concentrated. In Figure 14, the pattern of c7 (combined with p6 and p10) has close similarity with that of $\mathrm{p} 10$. The SLP difference triggered by $\mathrm{c} 7$ or $\mathrm{c} 10$ show a negative-over-positive tilted dipole with larger NAOI, deriving from the mutual promotion between two parameters. As for the $\mathrm{NAO}^{-}$, the SLP patterns of the 10 two-parameter combinations display an analogous structure in Figure 15. The positive pressure stretches over southern Greenland and western Europe, and the positive pressure center and the negative pressure center are symmetric about the $50 \mathrm{~N}$, exhibiting a typical dipole with no tilt. Compared with single parameters, two-parameter combinations have more potential to develop into NAO events.

Figures 16 and 17 depict the SLP difference for threeparameter combinations, with a range of $-5000(-4500)$ to 4500 (5500) Pa. All combinations form dipole pattern in the North Atlantic sector at a high intensity. The typical NAO mode proves that the parameter perturbation solved by CNOP-P approach can make the initial state evolve into NAO events, and the combination with three sensitive parameters can also cause the larger uncertainty than that of single parameters.

\section{Discussions and Conclusions}

Model parameter error is a critical factor that can trigger the uncertainty of the prediction result. Thus, the correction of parameters is beneficial to improve the prediction skill of numerical models. To provide direction on parameter correction, the sensitivity of the model parameter is investigated in this work. We adopt CESM to simulate the $\mathrm{NAO}$ and explore the impact of parameters that are sensitive to the NAO events. The research aims to find out the factors that are closely related to the atmospheric circulation of NAO. Our conclusions confirm the results of previous studies and also have some new discoveries.

From the result of the single parameter and parameter combinations, the parameter p22, which is related to solar irradiance, has a very significant impact on the simulation of both $\mathrm{NAO}^{+}$and $\mathrm{NAO}^{-}$in winter. It has been found that downward surface short-wave radiation has a strong relationship with the winter NAO index, even in the lowfrequency variability [52]. A recent article that examined the effects of solar activity cycles on winter NAO indicated that the mechanism of solar influence relies on the meridional temperature gradient, zonal winds, and the impact of ultraviolet radiation variability on heating rates in the tropical upper stratosphere [53]. The study compared constant solar radiation with time-varying solar irradiance, confirming the effect of sun on atmospheric heating and dynamic response. In addition, the research of Chiacchio et al. also found that cloud cover is a potential factor that can easily enhance the variability of the winter NAO [54]. As the parameter of deep convective cloud volume, p6 and p7 also display high NAO sensitivity in the cases of the single-parameter and parameter combinations. The above conclusions are consistent with the results of our work. If the model error, which caused by solar irradiance and volume of deep convective cloud, can be corrected, it would have a certain significance for improving the NAO prediction skills.

The precipitation is also a common predictor of NAO, with relative humidity as its direct relevant factor. In previous studies, the relationship between precipitation on the Iberian Peninsula and the NAO has been explored with simulating the NAOI change in DJFM (December, January, February, and March) [55]. In the single-parameter sensitivity analysis, p9 and p10, which denote the relative humidity of high stable cloud and low stable cloud, respectively, become the parameters that cause the maximum uncertainty of the prediction of $\mathrm{NAO}^{+}$. Moreover, p10 is also an important factor in triggering the maximum uncertainty of $\mathrm{NAO}^{-}$in a single parameter. The effects of greenhouse gas, sea surface temperature, and stratification variability on NAO variation have also been identified. In this paper, the parameters p1-p5 associated with greenhouse gases are covered in the group of potentially sensitive parameters. Some early studies have shown that increased greenhouse gas concentrations affect the stratospheric cycle and thus have an impact on climate change [56-58]. The concentration of greenhouse gases has increased with years in recent years. The main reason for the increase of $\mathrm{CO}_{2}$ concentration is the large-scale exploitation of fossil fuels, while the bio-fermentation process increases $\mathrm{CH}_{4}$ [59]. Although $\mathrm{N}_{2} \mathrm{O}$ and CFC-11 $\left(\mathrm{CCl}_{3} \mathrm{~F}\right)$ and CFC-12 $\left(\mathrm{CCl}_{2} \mathrm{~F}\right)$ are chemically inert in the troposphere, they can be photolyzed by solar radiation in the stratosphere, and the net increase rate is slower [60]. Our result is consistent with the work of the predecessors. 
In our work, the sensitivity of the single parameter and parameter combinations are analyzed and compared. We find that the combined influence of multiple parameters is more significant than the single parameters. The parameter perturbation causes a large difference between perturbation state and reference state, and it makes the basic state develop into a typical NAO mode at prediction time. It is also proved that CNOP-P is an effective method to conduct sensitivity analysis. Besides, the intelligence algorithms (PSO and CMA-ES) overcome the limitations of the adjoint model and result in a satisfying performance. Our work validates the related factors of the $\mathrm{NAO}$ and provides the correction direction of model parameters. In future work, we will optimize the sensitive parameters that would cause model errors and improve the prediction skill for the NAO events.

\section{Data Availability}

The data in our paper are all obtained by ourselves and all data are included in the supplementary materials.

\section{Conflicts of Interest}

The authors declare that there are no conflicts of interest regarding the publication of this paper.

\section{Acknowledgments}

The calculation of this work was performed on Tianhe-2. Thanks for the support of National Supercomputer Center in Guangzhou (NSCC-GZ). The authors also thank the help of Dai as an expert in the area of atmosphere. This study was supported by the Fundamental Research Funds for the Central Universities (grant no. 22120190207), the Key Project Fund of Shanghai 2020 "Science and Technology Innovation Action Plan" for Social Development (grant no. 20dz1200702), and National Natural Science Foundation of China (grant no. 42075141).

\section{References}

[1] G. Walker, "World weather," Quarterly Journal of the Royal Meteorological Society, vol. 54, no. 226, pp. 79-87, 1928.

[2] J. W. Hurrell, "Decadal trends in the north atlantic oscillation: regional temperatures and precipitation," Science, vol. 269, no. 5224 , pp. 676-679, 1995.

[3] J. W. Hurrell, "Influence of variations in extratropical wintertime teleconnections on northern hemisphere temperature," Geophysical Research Letters, vol. 23, no. 6, pp. 665-668, 2013.

[4] J. Sillmann and M. Croci-Maspoli, "Present and future atmospheric blocking and its impact on european mean and extreme climate," Geophysical Research Letters, vol. 36, no. 10, pp. 92-103, 2009.

[5] M. Irina, M. Olivia, C. Clment, and G. David, "Changes in the odds of extreme events in the atlantic basin depending on the position of the extra tropical jet," Geophysical Research Letters, vol. 39, no. 22, 2012.

[6] C. Ouzeau and S. Douville, "European cold winter 2009-2010: how unusual in the instrumental record and how re- producible in the arpege-climat model?" Geophysical Research Letters, vol. 38, no. 11, 2011.

[7] C. Wang, H. Liu, and S.-K. Lee, "The record-breaking cold temperatures during the winter of 2009/2010 in the northern Hemisphere," Atmospheric Science Letters, vol. 11, no. 3, pp. 161-168, 2010.

[8] J. W. Hurrell, Y. Kushnir, G. Ottersen, and M. Visbeck, "The north atlantic oscillation: climatic significance and environmental impact," EOS Transactions American Geophysical Union, vol. 84, no. 8, p. 73, 2013.

[9] J. J. B. S. Lee and S. B. Feldstein, "Synoptic view of the north atlantic oscillation," Journal of the Atmospheric Sciences, vol. 61, no. 2, pp. 121-144, 2002.

[10] F. Hansen, R. J. Greatbatch, G. Gollan, T. Jung, and A. Weisheimer, "Remote control of north atlantic oscillation predictability via the stratosphere," Quarterly Journal of the Royal Meteorological Society, vol. 143, no. 703, pp. 706-719, 2017.

[11] B. Yu and H. Lin, "Tropical atmospheric forcing of the wintertime north atlantic oscillation," Journal of Climate, vol. 29, no. 5, pp. 1755-1772, 2016.

[12] V. Bucha, "Geomagnetic activity and the north atlantic oscillation," Studia Geophysica et Geodaetica, vol. 58, no. 3, pp. 461-472, 2014.

[13] D. Luo, J. Cha, and S. B. Feldstein, "Weather regime transitions and the interannual variability of the north atlantic oscillation. part i: a likely connection," Journal of the Atmospheric Sciences, vol. 69, no. 8, pp. 2329-2346, 2012.

[14] D. Luo, J. Cha, and S. B. Feldstein, "Weather regime transitions and the interannual variability of the north atlantic oscillation. part i: a likely connection," Journal of the Atmospheric Sciences, vol. 69, no. 8, pp. 2347-2363, 2011.

[15] H. Pohlmann, D. M. Smith, M. A. Balmaseda et al., "Predictability of the mid-latitude atlantic meridional overturning circulation in a multi-model system," Climate Dynamics, vol. 41, no. 3-4, pp. 775-785, 2013.

[16] S. Müller, P. D. Pietro, A. Storto, S. Dobricic, A. Alessandri, and A. Cherchi, Re-analyses in the Global Ocean at CMCCINGV: Examples and Applications, Earth Prints, Washingston, DC, USA, 2010.

[17] W. Zhiyuan, L. I. Yao, L. Bin, and L. Jian, "Global climate internal variability in a 2000-year control simulation with community earth system model (CESM)," Chinese Geographical Science, vol. 25, no. 3, pp. 263-273, 2015.

[18] J. J. Nauw, H. A. Dijkstra, and E. P. Chassignet, "Frictionally induced asymmetries in wind-driven flows," Journal of Physical Oceanography, vol. 34, no. 9, pp. 2057-2072, 2004.

[19] O. Martínez-Alvarado, "Implications of model error for numerical climate prediction," Nonlinear Processes in Geophysics Discussions, vol. 1, no. 1, pp. 131-153, 2014.

[20] Y. Y. Luo and S. B. F. Dai, "The positive north atlantic oscillation with downstream blocking and middle east snowstorm: the large-scale environment," Journal of Climate, vol. 28, no. 16, Article ID 63986418, 2015.

[21] K. Steffens, M. Larsbo, J. Moeys, N. Jarvis, and E. Lewan, "Predicting pesticide leaching under climate change: importance of model structure and parameter uncertainty," Agriculture, Ecosystems \& Environment, vol. 172, no. 6, pp. 24-34, 2013.

[22] S. Zaehle, S. Sitch, B. Smith, and F. Hatterman, "Effects of parameter uncertainties on the modeling of terrestrial biosphere dynamics," Global Biogeochemical Cycles, vol. 19, no. 3, Article ID GB3020, 2005. 
[23] X. Yin, B. Wang, J. Liu, and X. Tan, "Evaluation of conditional non-linear optimal perturbation obtained by an ensemblebased approach using the lorenz-63 model," Tellus A: Dynamic Meteorology and Oceanograph, vol. 66, 2014.

[24] D. M. Hamby, "A review of techniques for parameter sensitivity analysis of environmental models," Environmental Monitoring and Assessment, vol. 32, no. 2, pp. 135-154, 1994.

[25] C.-S. Zhan, X.-M. Song, J. Xia, and C. Tong, "An efficient integrated approach for global sensitivity analysis of hydrological model parameters," Environmental Modelling \& Software, vol. 41, pp. 39-52, 2013.

[26] V. Griensven, G. Meixner, and D. Bishop, "A global sensitivity analysis tool for the parameters of multi-variable catchment models," Journal of Hydrology, vol. 324, no. 1, pp. 10-23, 2006.

[27] C. F. Ma, L. Xin, and S. G. Wang, "A global sensitivity analysis of soil parameters associated with backscattering using the advanced integral equation model," IEEE Transactions on Geoscience Remote Sensing, vol. 53, no. 10, pp. 5613-5623, 2015.

[28] J. Domke and D. Wang, "Runtime tracing of the community earth system model: feasibility study and benefits," Procedia Computer Science, vol. 9, no. 11, pp. 1950-1958, 2012.

[29] C. Deser, J. W. Hurrell, and A. S. Phillips, "The role of the north atlantic oscillation in european climate projections," Climate Dynamics, vol. 49, no. 9-10, pp. 3141-3157, 2017.

[30] Y.-O. Kwon, A. Camacho, C. Martinez, and H. Seo, "North atlantic winter eddy-driven jet and atmospheric blocking variability in the community earth system model version 1 large ensemble simulations," Climate Dynamics, vol. 51, no. 910, pp. 3275-3289, 2018.

[31] M. Mu, W. Duan, Q. Wang, and R. Zhang, "An extension of conditional nonlinear optimal perturbation approach and its applications," Nonlinear Processes in Geophysics, vol. 17, no. 2, pp. 211-220, 2010.

[32] Y. Yu, M. Mu, and W. Duan, "Does model parameter error cause a significant "spring predictability barrier" for el niño events in the zebiak-cane model?" Journal of Climate, vol. 25, no. 4, pp. 1263-1277, 2012.

[33] G. Sun and M. Mu, "Response of a grassland ecosystem to climate change in a theoretical model," Advances in Atmospheric Sciences, vol. 28, no. 6, pp. 1266-1278, 2011.

[34] G. Sun and M. Mu, "Responses of soil carbon variation to climate variability in China using the lpj model," Theoretical Applied Climatology, vol. 110, no. 1-2, pp. 143-153, 2012.

[35] G. Sun, P. Fei, and M. Mu, "Uncertainty assessment and sensitivity analysis of soil moisture based on model parameters results from four regions in China," Journal of $\mathrm{Hy}$ drology, vol. 555, 2017.

[36] S. Yuan, H. Zhang, M. Li, and B. Mu, "Cnop-p-based parameter sensitivity for double-gyre variation in roms with simulated annealing algorithm," Journal of Oceanology and Limnology, vol. 37, no. 3, pp. 957-967, 2019.

[37] G. Sun and M. Mu, "Assessing the characteristics of net primary production due to future climate change and co 2 under rcp4.5 in China," Ecological Complexity, vol. 34, pp. 58-68, 2018.

[38] B. Wang, P. Zhang, Z. Huo, and Q. Qi, "The sensitivity analysis of a lake ecosystem with the conditional nonlinear optimal perturbation method," Advances in Meteorology, vol. 20127 pages, 2012.

[39] B. Mu, S. Wen, S. Yuan, and H. Li, "PPSO: PCA based particle swarm optimization for solving conditional nonlinear optimal perturbation," Computers \& Geosciences, vol. 83, pp. 65-71, 2015.
[40] Q. Zheng, Z. Yang, J. Sha, and J. Yan, "Conditional nonlinear optimal perturbations based on the particle swarm optimization and their applications to the predictability problems," Nonlinear Processes in Geophysics, vol. 24, no. 1, pp. 101-112, 2017.

[41] S. Yuan, J. Feng, J. Yan, and B. Mu, "A parallel sensitive area selection-based particle swarm optimization algorithm for fast solving cnop," in Proceedings of the International Conference on Neural Information Processing, Istanbul, Turkey, November 2015.

[42] C. Li, P. Heinemann, and R. Sherry, "Neural network and bayesian network fusion models to fuse electronic nose and surface acoustic wave sensor data for apple defect detection," Sensors and Actuators B: Chemical, vol. 125, no. 1, pp. 301-310, 2007.

[43] Y. H. Jia, Y. R. Zhou, Y. Lin, W. J. Yu, Y. Gao, and L. Lu, “A distributed cooperative co-evolutionary cma evolution strategy for global optimization of large-scale overlapping problem," IEEE Access, vol. 7, 2019.

[44] E. Taci, O. Gkalp, and A. Uur, "Development of a novel feature weighting method using cma-es optimization," in Proceedings of the 2018 26th Signal Processing and Communications Applications Conference, pp. 1-4, Izmir, Turkey, May 2018.

[45] S. M. Larson, D. J. Vimont, A. C. Clement, and B. P. Kirtman, "How momentum coupling affects SST variance and largescale pacific climate variability in CESM," Journal of Climate, vol. 31, no. 7, pp. 2927-2944, 2018.

[46] A. Merrifield, F. Lehner, S.-P. Xie, and C. Deser, "Removing circulation effects to assess central us land-atmosphere interactions in the cesm large ensemble: isolating land surface feedback in cesm," Geophysical Research Letters, vol. 44, 2017.

[47] T. Glotfelty and Y. Zhang, "Impact of future climate policy scenarios on air quality and aerosol-cloud interactions using an advanced version of cesm/cam5: part ii. future trend analysis and impacts of projected anthropogenic emissions," Atmospheric Environment, vol. 152, pp. 531-552, 2017.

[48] Q. Liu, "On the definition and persistence of blocking," Tellus A, vol. 46, no. 3, pp. 286-298, 1994.

[49] J. Kennedy and R. Eberhart, "Article swarm optimization," in Proceedings of the ICNN'95-International Conference on Neural Networks, pp. 1942-1948, Perth, Australia, December 1995.

[50] V. Vaiciulis, J. Vencloviene, A. Tamosiunas, R. Radisauskas, D. Luksiene, and G. Bernotiene, "The influence of the north atlantic oscillation index on arterial blood pressure," European Journal of Public Health, vol. 28, 2018.

[51] M. Beniston and P. Jungo, "Shifts in the distributions of pressure, temperature and moisture and changes in the typical weather patterns in the Alpine region in response to the behavior of the north atlantic oscillation," Theoretical and Applied Climatology, vol. 71, no. 1-2, pp. 29-42, 2002.

[52] D. Pozo-Vzquez, J. Tovar-Pescador, S. R. Gmiz-Fortis, M. J. Esteban-Parra, and Y. Castro-Dez, "Nao and solar radiation variability in the european north atlantic region," Geophysical Research Letters, vol. 31, no. 5, pp. 325-341, 2004.

[53] M. B. Andrews, J. R. Knight, and L. J. Gray, "A simulated lagged response of the north atlantic oscillation to the solar cycle over the period 1960-2009," Environmental Research Letters, vol. 10, no. 5, pp. 321-378, 2015.

[54] M. Chiacchio, T. Ewen, M. Wild, and E. Arabini, "Influence of climate shifts on decadal variations of surface solar radiation in Alaska," Journal of Geophysical Research, vol. 115, Article ID D00D22, 2010. 
[55] C. Rodríguez-Puebla and S. Nieto, "Trends of precipitation over the iberian peninsula and the north atlantic oscillation under climate change conditions," International Journal of Climatology, vol. 30, 2010.

[56] D. T. Shindell, R. L. Miller, G. A. Schmidt, and L. Pandolfo, "Simulation of recent northern winter climate trends by greenhouse-gas forcing," Nature, vol. 399, no. 6735, pp. 452-455, 1999.

[57] M. P. Hoerling, J. W. Hurrell, and T., T. Xu, "Tropical origins for recent north atlantic climate change," Science, vol. 292, no. 5514, pp. 90-92, 2001.

[58] E. K. Schneider, L. Bengtsson, and Z.-Z. Hu, "Forcing of northern hemisphere climate trends," Journal of the Atmospheric Sciences, vol. 60, no. 12, pp. 1504-1521, 2003.

[59] X. Hao, F. J. Larney, C. Chang, G. R. Travis, C. K. Nichol, and E. Bremer, "The effect of phosphogypsum on greenhouse gas emissions during cattle manure composting," Journal of Environmental Quality, vol. 34, no. 3, pp. 774-781, 2005.

[60] D. J. Hofmann, J. H. Butler, E. J. Dlugokencky et al., "The role of carbon dioxide in climate forcing from 1979 to 2004: introduction of the annual greenhouse gas index," Tellus Series B-Chemical Physical Meteorology, vol. 58, no. 5, pp. 614-619, 2010 . 